\title{
A Systematic Review on the Association Between Tear Film Metrics and Higher Order Aberrations in Dry Eye Disease and Treatment
}

\author{
Jess Rhee - Tommy Chung-Yan Chan · Sharon She-Wan Chow • \\ Antonio Di Zazzo · Takenori Inomata · Kendrick Co Shih • \\ Louis Tong
}

Received: July 29, 2021 / Accepted: October 29, 2021 / Published online: November 11, 2021

(C) The Author(s) 2021

\section{ABSTRACT}

We systematically reviewed published research on dry eye disease and its association with higher order aberrations (HOAs). The purpose of this review was to first determine if an association between tear film metrics and HOAs exists

J. Rhee

Schulich School of Medicine and Dentistry, Western University, London, ON, Canada

T. C.-Y. Chan

Department of Ophthalmology, Hong Kong Sanatorium and Hospital, Hong Kong, SAR, China

T. C.-Y. Chan · S. S.-W. Chow · K. C. Shih (ه) Department of Ophthalmology, Li Ka Shing Faculty of Medicine, The University of Hong Kong, 301B Cyberport 4, 100 Cyberport Road, Pokfulam, Hong Kong, SAR, China

e-mail: kcshih@hku.hk

A. Di Zazzo

Ophthalmology Operative Complex Unit,

University Campus Bio-Medico, 00128 Rome, Italy

T. Inomata

Department of Ophthalmology, Juntendo

University Graduate School of Medicine, Tokyo

1130033, Japan

L. Tong

Cornea and External Eye Disease Service, Singapore

National Eye Centre, Singapore, Singapore

L. Tong

Ocular Surface Research Group, Singapore Eye

Research Institute, Singapore, Singapore and second to determine if the treatment of dry eyes can improve tear film metrics and HOAs together. A search was conducted in Entrez PubMed on 25 April 2021 using the keywords "higher order aberrations" and "dry eye". The initial search yielded 61 articles. After publications were restricted to only original articles measuring HOA outcomes in patients with dry eye, the final yield was 27 relevant articles. Of these 27 papers, 12 directly looked at associations and correlations between dry eyes and HOA parameters. The remaining 15 studies looked at dry eye interventions and HOA outcomes and parameters. There is clear evidence demonstrating that dry eyes and HOAs have an association, and that the tear film is one of the most important factors in this relationship. There is also a direct correlation between tear film metrics and HOAs. Improvements in HOAs with dry eye interventions provide further evidence to support the intricate relationship between the two. Despite the clear association between HOAs and dry eye disease, further research is still required in the realm of clinical application as dry eye interventions vary depending on many factors, including patient severity and eye drop viscosity.

Keywords: Dry eye disease; Dry eye treatment; Higher order aberrations; Tear film 


\section{Key Summary Points}

The rationale for this review was to determine the association between tear film metrics and higher order aberrations (HOAs), and to also determine if treatment of dry eyes improves tear film metrics and HOAs.

The literature was systematically reviewed, in that the total of 60 search hits with our key terms were then curated by the authors on the basis of original research and a focus on tear film metrics and HOA parameters, leading to a total of 26 relevant articles for this review.

This review has shown clear associations and correlations between dry eyes and HOA parameters.

Improvements in HOAs with dry eye interventions provide further evidence to support the intricate relationship between the two.

However, despite the clear association between HOAs and dry eye disease, further research is still required in the realm of clinical application as many factors such as patient severity and eye drop viscosity can impact clinical outcomes.

\section{INTRODUCTION}

The formation of optical images on the retina marks the beginning stages of vision processing [1]. However, there are many possible avenues of error either through diffraction, errors of focus, and wavefront aberrations [1, 2]. When optical systems are in "perfect" settings, rays emanating from an object are refracted to converge to the unique image point as expected from Gaussian theory [1]. However, when the system suffers from wavefront aberrations, the refracted rays no longer converge to the expected unique image point [1]. Therefore, the image is defocused or can be distorted. In particular, higher order aberrations (HOAs) have been of growing interest. Although most HOAs do not cause any noticeable degradation in visual acuity below 20/20 vision, laser and other refractive surgeries (including cataract-related ones) have left patients with symptomatic complaints of dry eye related to HOAs [1, 3].

Aberrometers are able to measure wavefront aberrations, i.e., the distance between an actual wavefront and the ideal spherical or "reference" wavefront on the ideal image point [1]. One of the most common designs is the Hartmann-Shack aberrometer which shoots a narrow beam of light into the eye to produce a reflection [1]. If the eye is free of aberrations, this ideally would result in a parallel ray leaving the eye and the existing wavefronts strike a Hartmann-Shack wavefront sensor comprising an array of identical converging micro lenses [1]. The overall ideal result is image points reflecting directly in a regular grid pattern. However, when aberrations are present, these image points are displaced by an amount and direction depending on the aberration, resulting in an irregular grid pattern [1].

An important factor that affects HOAs includes the tear film [2, 4, 5]. The tear film is the first refracting surface that light comes into contact with and thus has a crucial role in maintaining a smooth corneal surface $[2,4,5]$. Changes to the tear film thickness and regularity can introduce additional aberrations [4]. As the eyes remain open, the tear film eventually breaks up and this also causes irregularity and increased aberrations [2, 4, 5]. Dry eye can be defined as a tear deficiency disorder of the ocular surface [6]. Several studies have demonstrated that disruptions of tear film caused by dry eye increase HOAs compared with normal eyes which can result in impaired visual function and optical quality [4, 6-9].

One way to address the symptoms and reported impaired optical quality of dry eyes is to use lubricating eye drops that have been shown to improve optical quality and significantly decrease aberrations associated with irregular tear films and dry eyes [2, 10-12]. Another strategy or intervention for dry eyes is 
punctal plugs to block the puncta allowing for increased tear fluid accumulation, thus keeping the tear film intact [13].

Dry eyes are one of the most common ocular disorders and a complication of many common laser and refractive surgeries, including cataract surgeries [1, 2, 13]. Therefore, it is important to understand any potential association or pathophysiology of HOAs and dry eyes, as well as whether interventions that improve dry eyes reduce HOA severity.

\section{METHODS}

To determine the primary literature available focussing on HOAs and dry eye association as well as dry eye interventions and HOA outcomes, the following search strategy was used in Entrez PubMed on 25 April 2021. The key terms used were "higher order aberrations" and "dry eyes" with an "AND" between each keyword for a keyword search string of "higher order aberrations AND dry eyes". No filters were set. This resulted in a total search hit of 61 articles. Figure 1 illustrates the screening process in a flowchart diagram. The articles were curated to determine whether they were original research articles; this excluded 13 articles as they were review articles. The second curation was based on subject relevance-if a paper did not look into dry eyes and HOAs as an outcome parameter, it would not be included. The second curation excluded 21 papers leaving a total of 27 relevant articles for this review (Fig. 1).

This article is based on previously conducted studies and does not contain any new studies with human participants or animals performed by any of the authors.

\section{RESULTS}

The search criteria and curation process yielded 27 relevant papers that looked at the primary outcome of HOAs in patients with dry eye. Of these 27 papers, 12 directly looked at any association and correlation of dry eyes and HOAs and HOA parameters. The remaining 15 studies looked at dry eye interventions and HOA outcomes and parameters. The main results of each paper are summarized in Tables 1 and 2 .

\section{HOAs and Dry Eye Association}

The first paper retrospectively reviewed patients with chronic ocular graft-versus-host disease (GVHD) after hematopoietic stem cell transplantation (HSCT) with dry eye disease [14]. Dry eye is the main and most common phenotype for GVHD in the eye. Although chronic ocular GVHD does not typically lead to visual loss and recipients generally have good visual acuity, the vision-related quality of life is impaired. The total and anterior HOAs at $4-\mathrm{mm}$ and $6-\mathrm{mm}$ diameters in patients with GVHD were significantly higher compared to both non-GVHD and healthy controls. Total and anterior spherical aberrations (SA) at both $4-\mathrm{mm}$ and $6-\mathrm{mm}$ diameters were significantly lower in patients with GVHD compared to healthy controls. Total and anterior coma aberrations at 4-mm diameters were significantly higher in patients with GVHD compared to patients without GVHD while total coma aberrations at 6-mm were significantly higher in GVHD compared to healthy controls. When the HOAs in the same patients were analyzed and compared to their contralateral eyes, patients with GVHD had significantly higher HOAs compared to patients without GVHD. A significantly positive correlation was found between HOAs and visual acuity (logMAR) as well. Although dry eye is the most common phenotype for GVHD in the eye, the increase in HOAs revealed could also be attributed to the inflammation on the ocular surface as opposed to a direct effect of dry eyes.

One group prospectively assessed HOAs in patients with normal and dry eyes [4]. The group was able to demonstrate that HOAs were significantly higher in patients with dry eye for both 4-mm and 6-mm diameters. This included coma, spherical, and total aberrations for both 4- and 6-mm diameters. Their quantitative analysis of the Hartmann-Shack images showed a significant change in the wavefront patterns in dry eyes compared to normal eyes. Specifically, these corresponded to reduced optical path length, which indicated relative thinning 


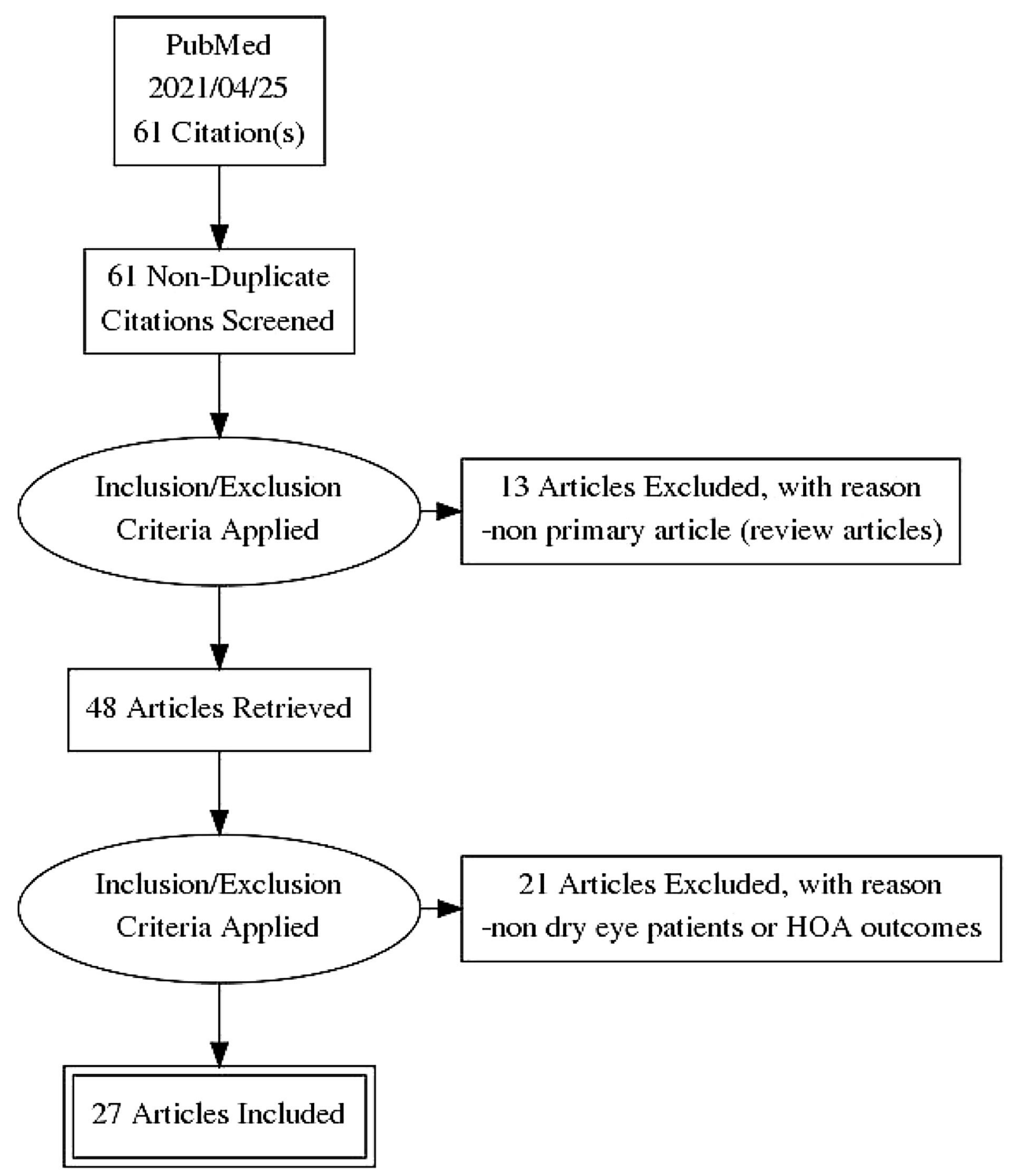

Fig. 1 PRISMA (Preferred Reporting Items for Systematic Reviews and Meta-analyses) flowchart illustrating the selection process of articles 


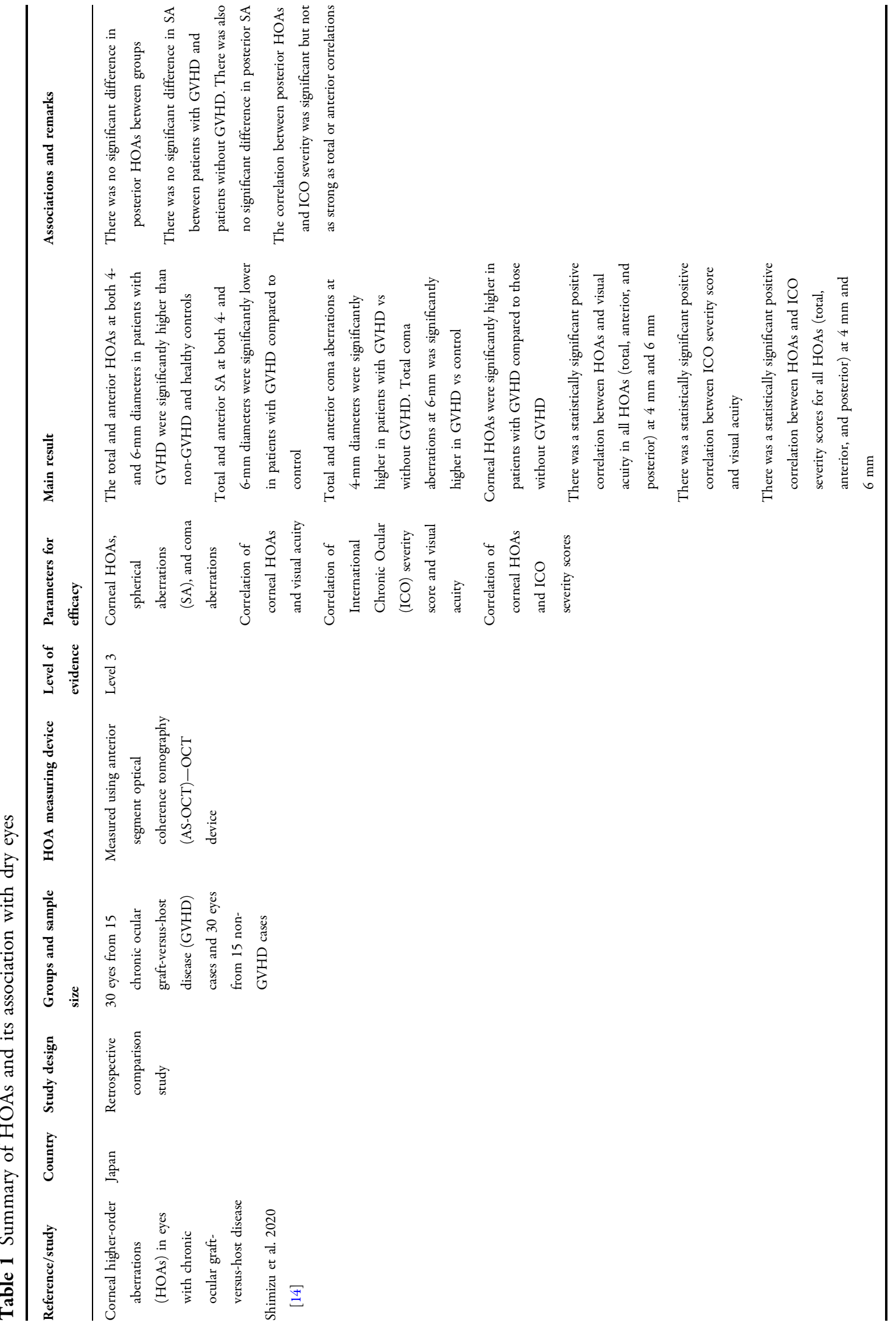




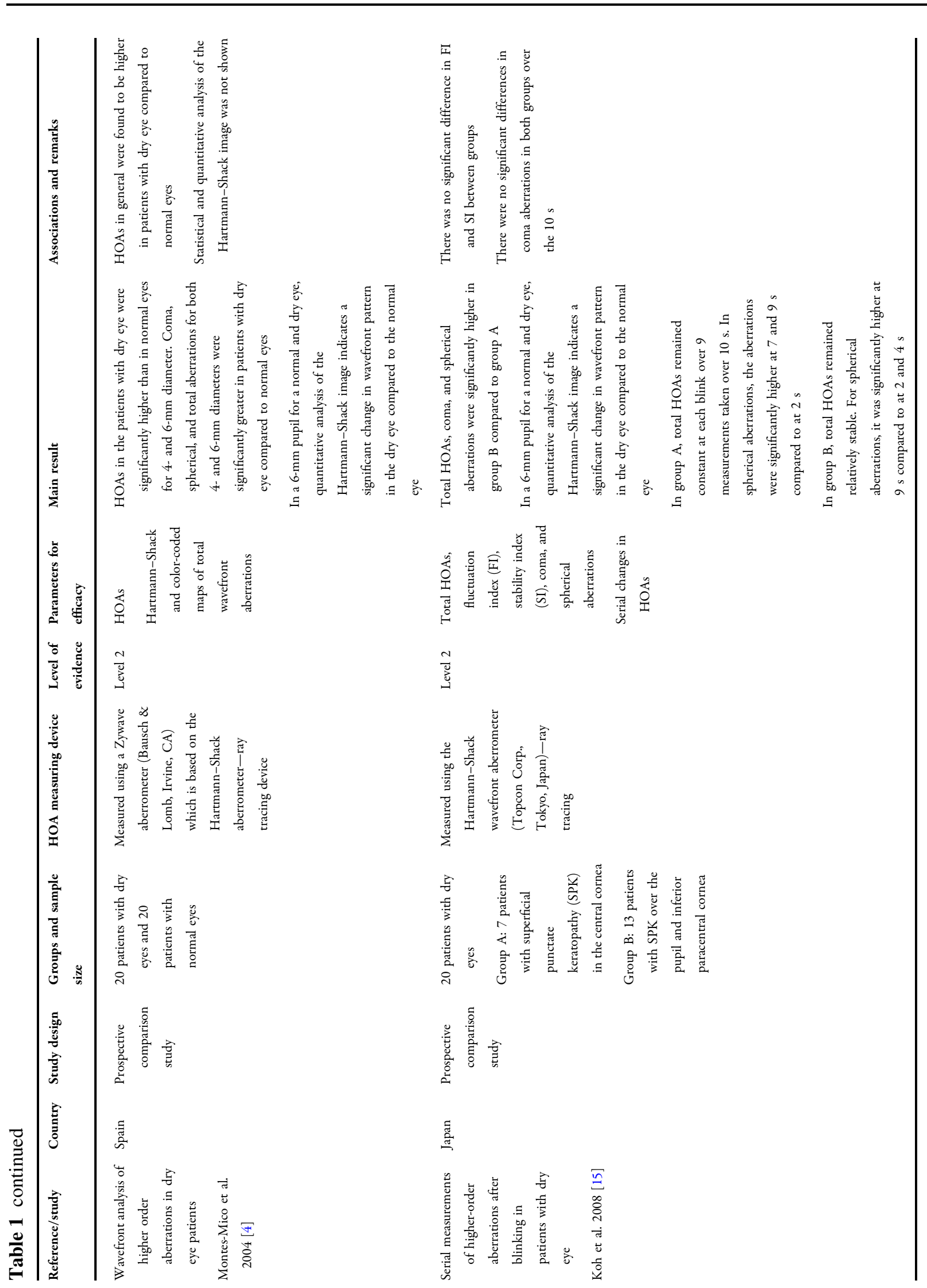




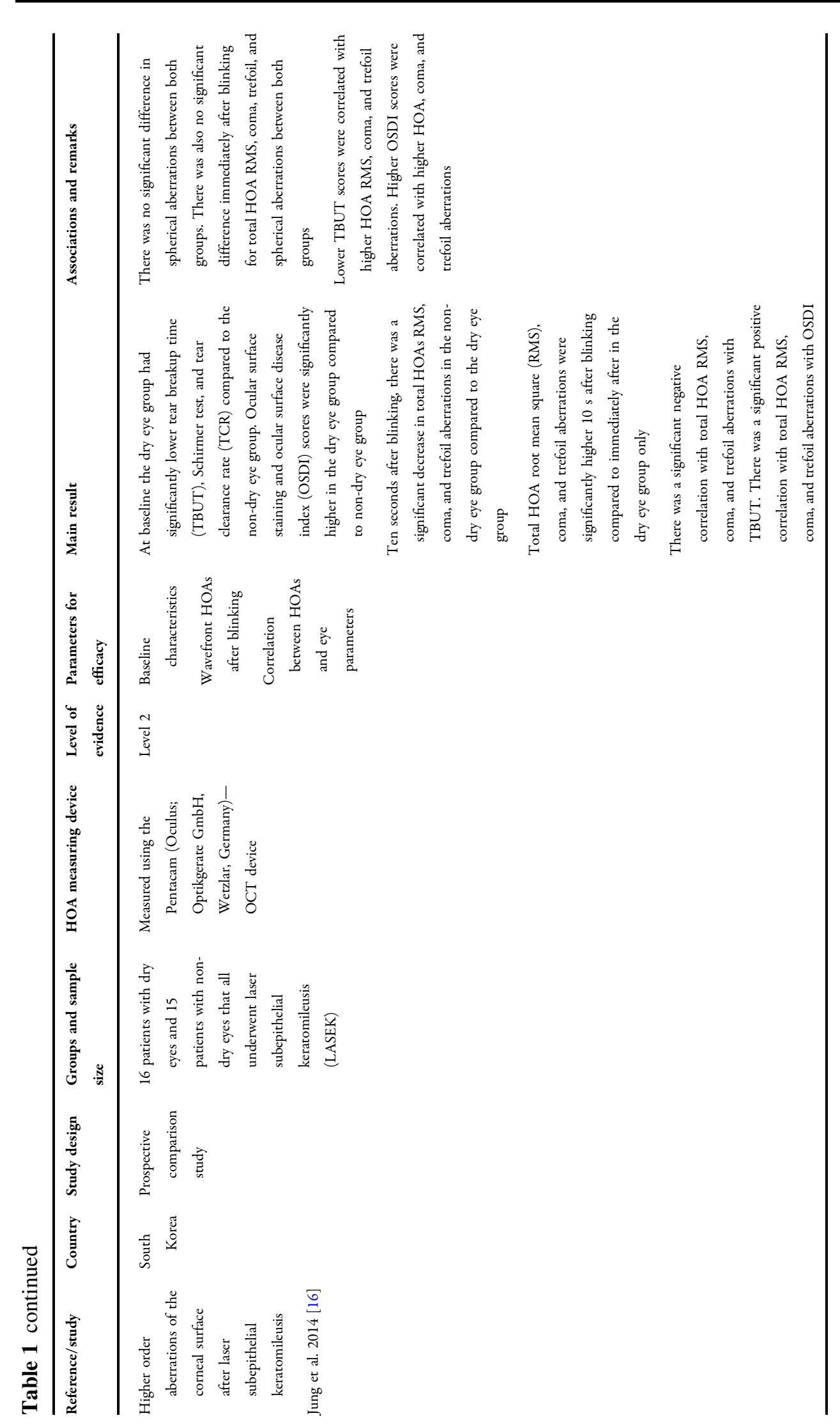


42

Ophthalmol Ther (2022) 11:35-67

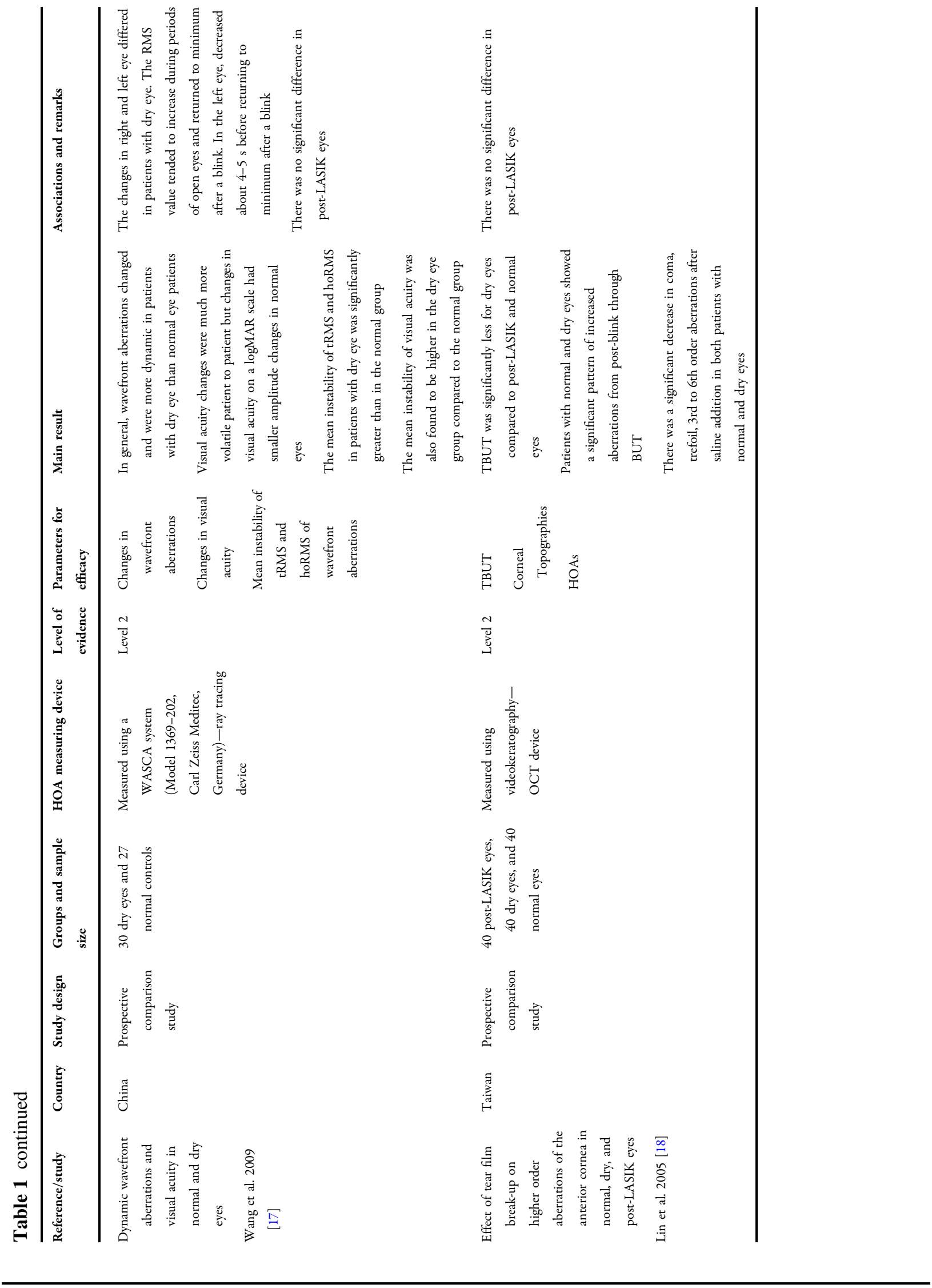

么 Adis 


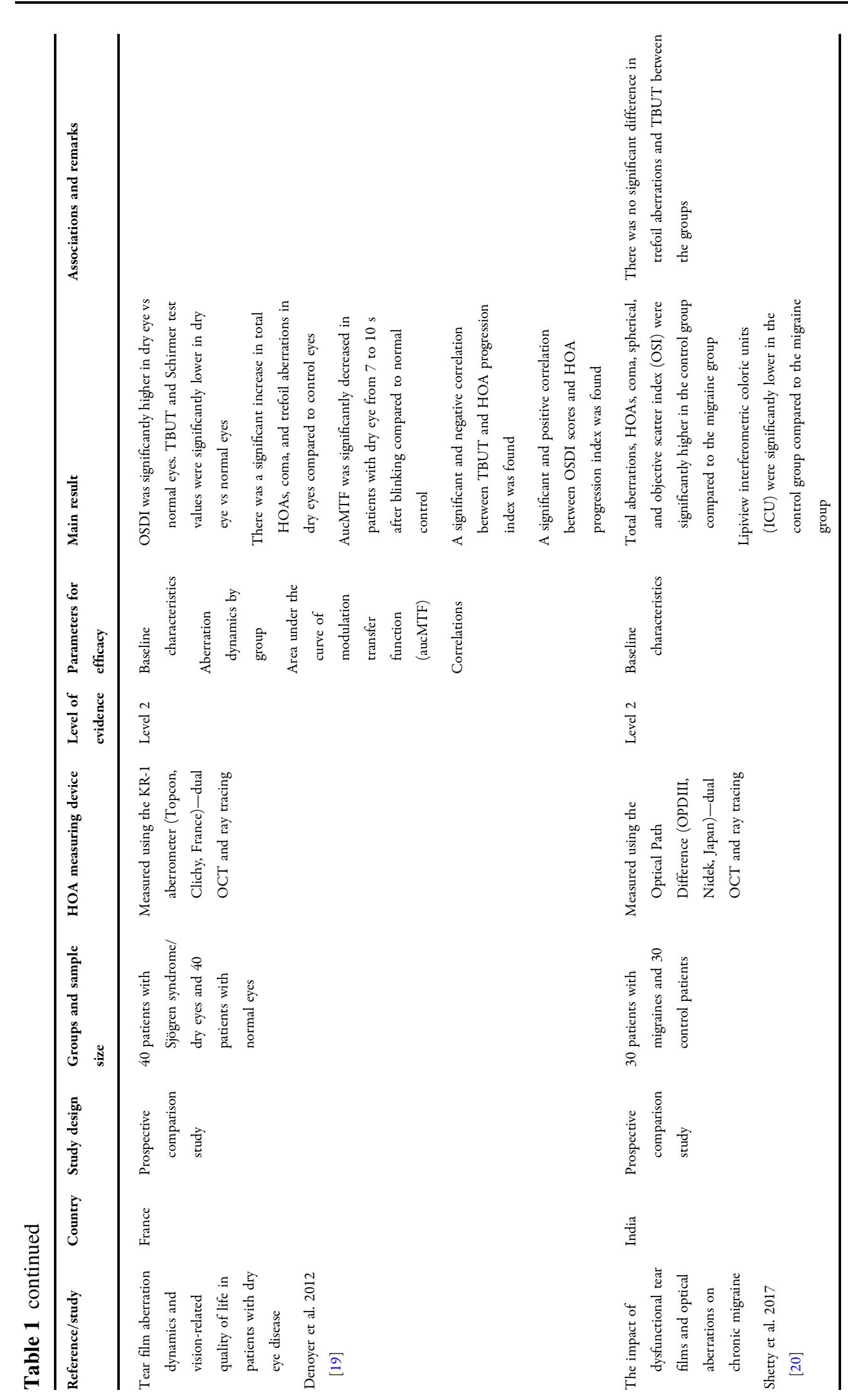




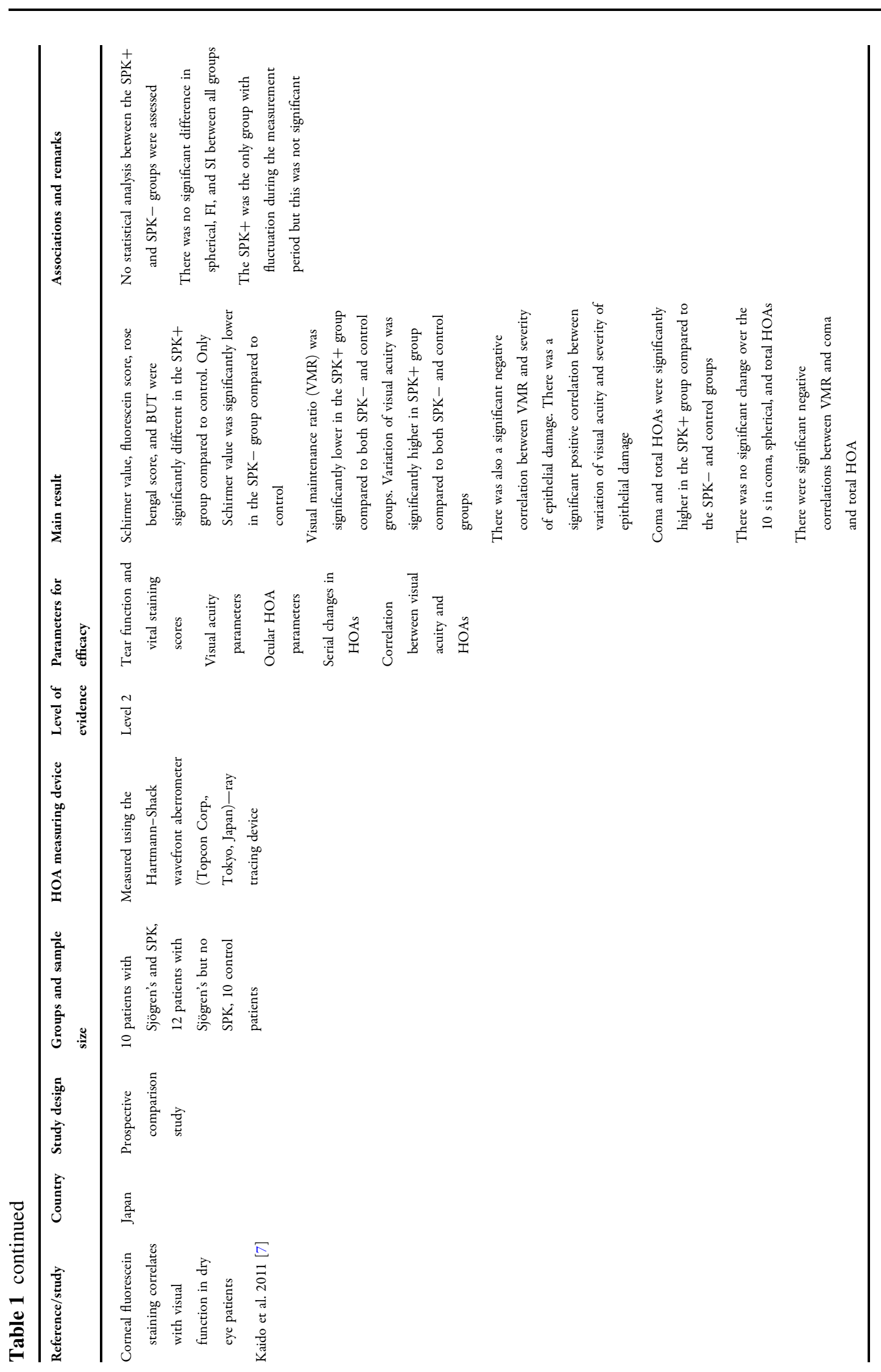




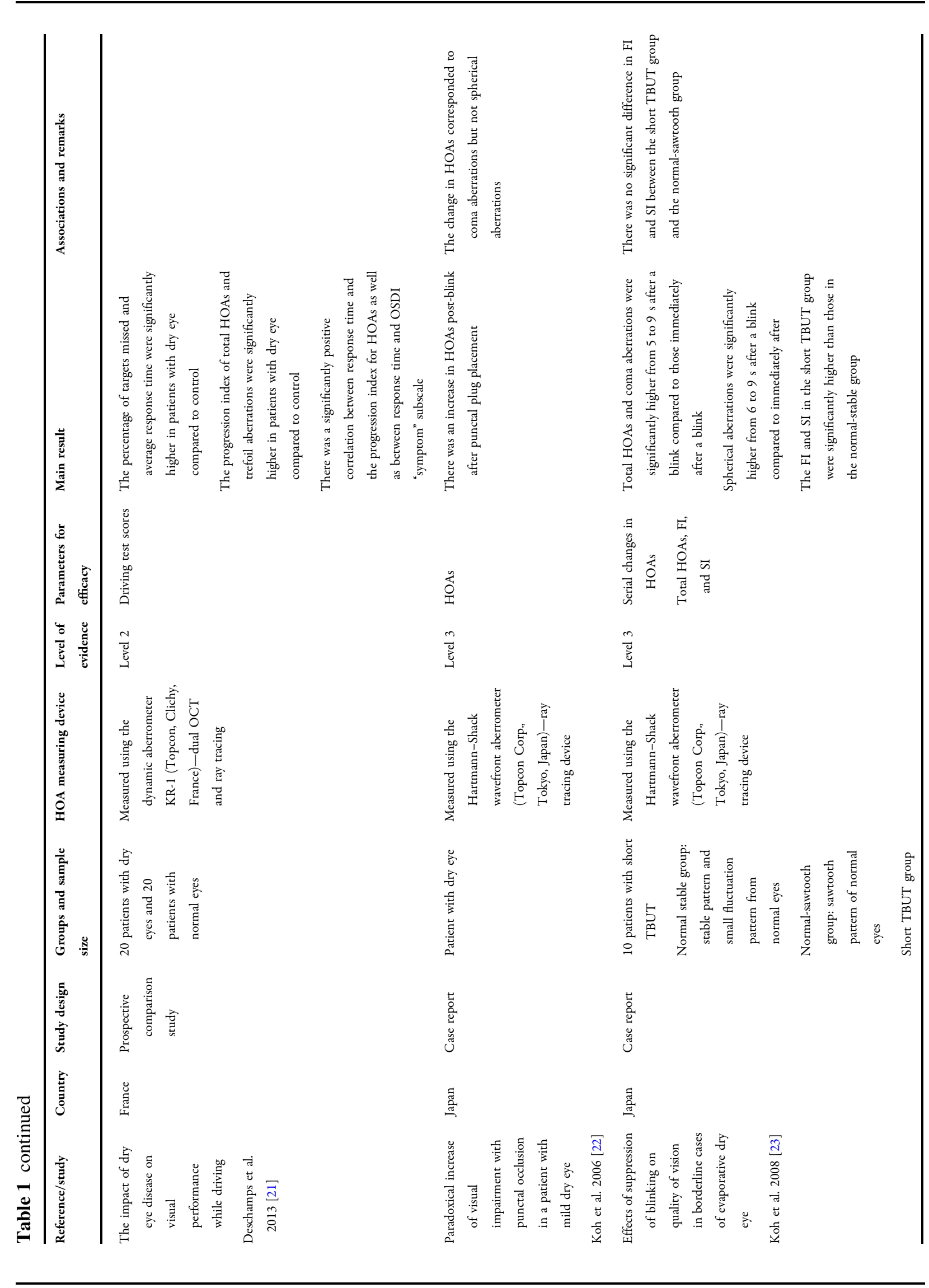




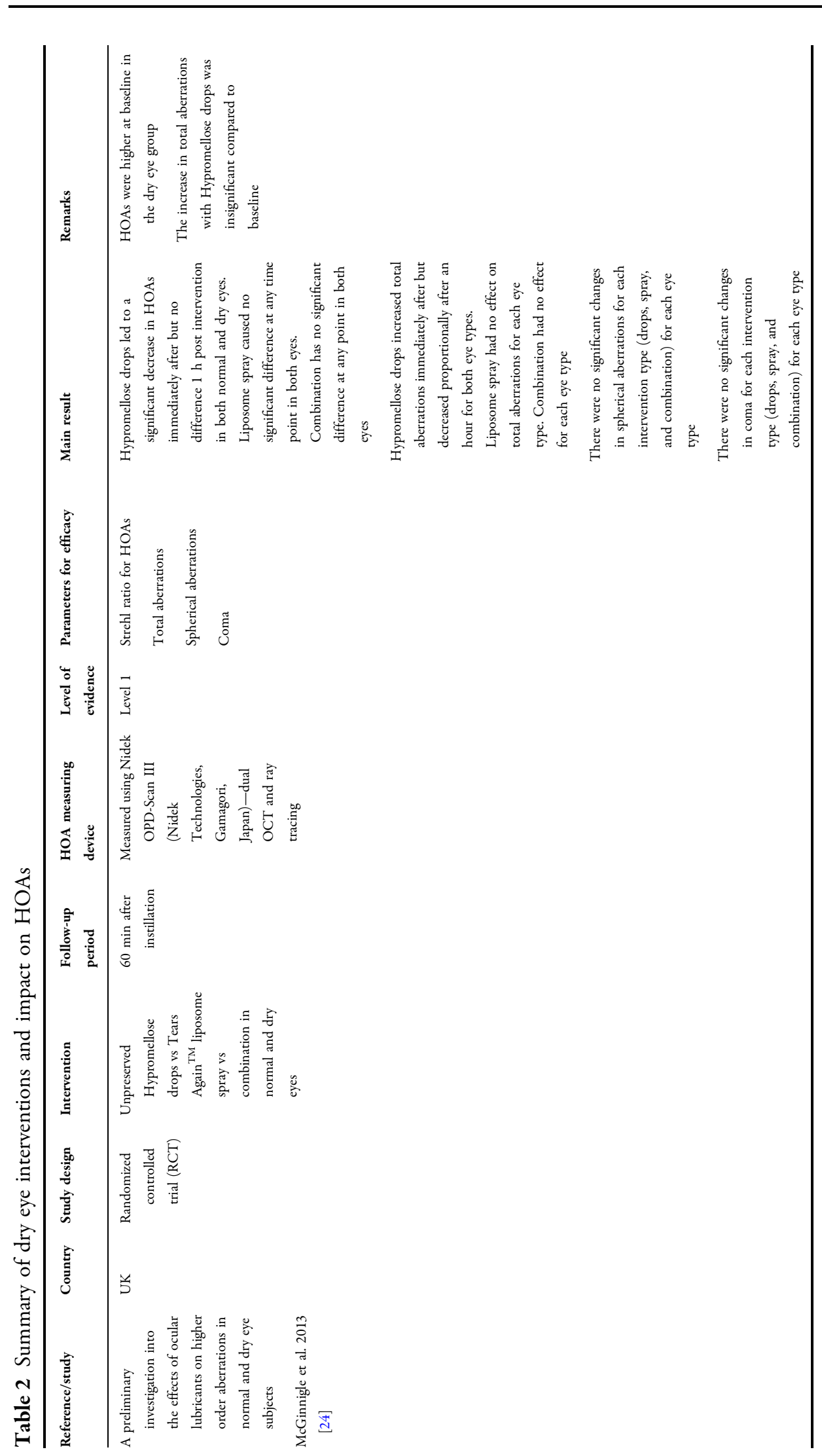




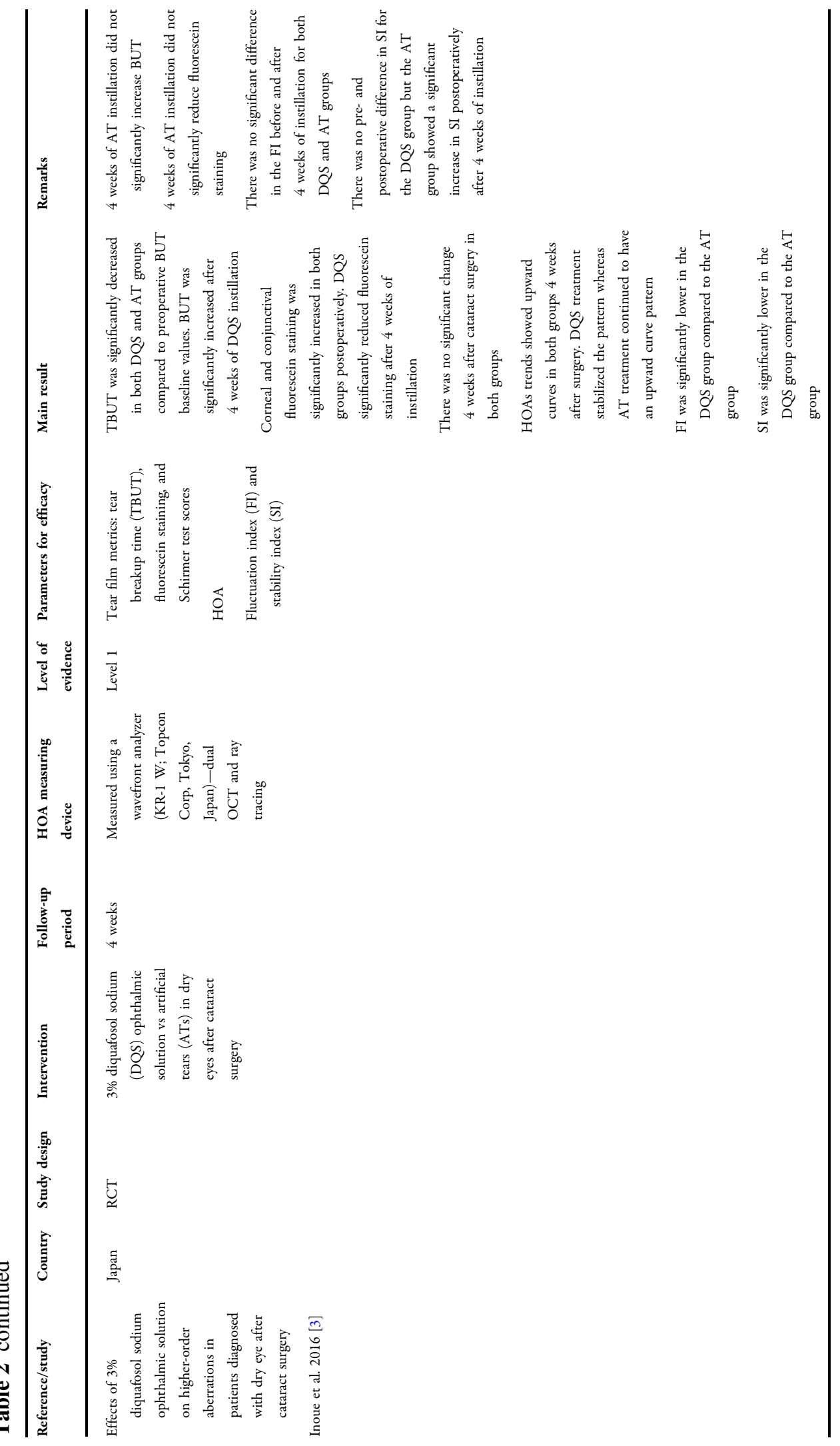




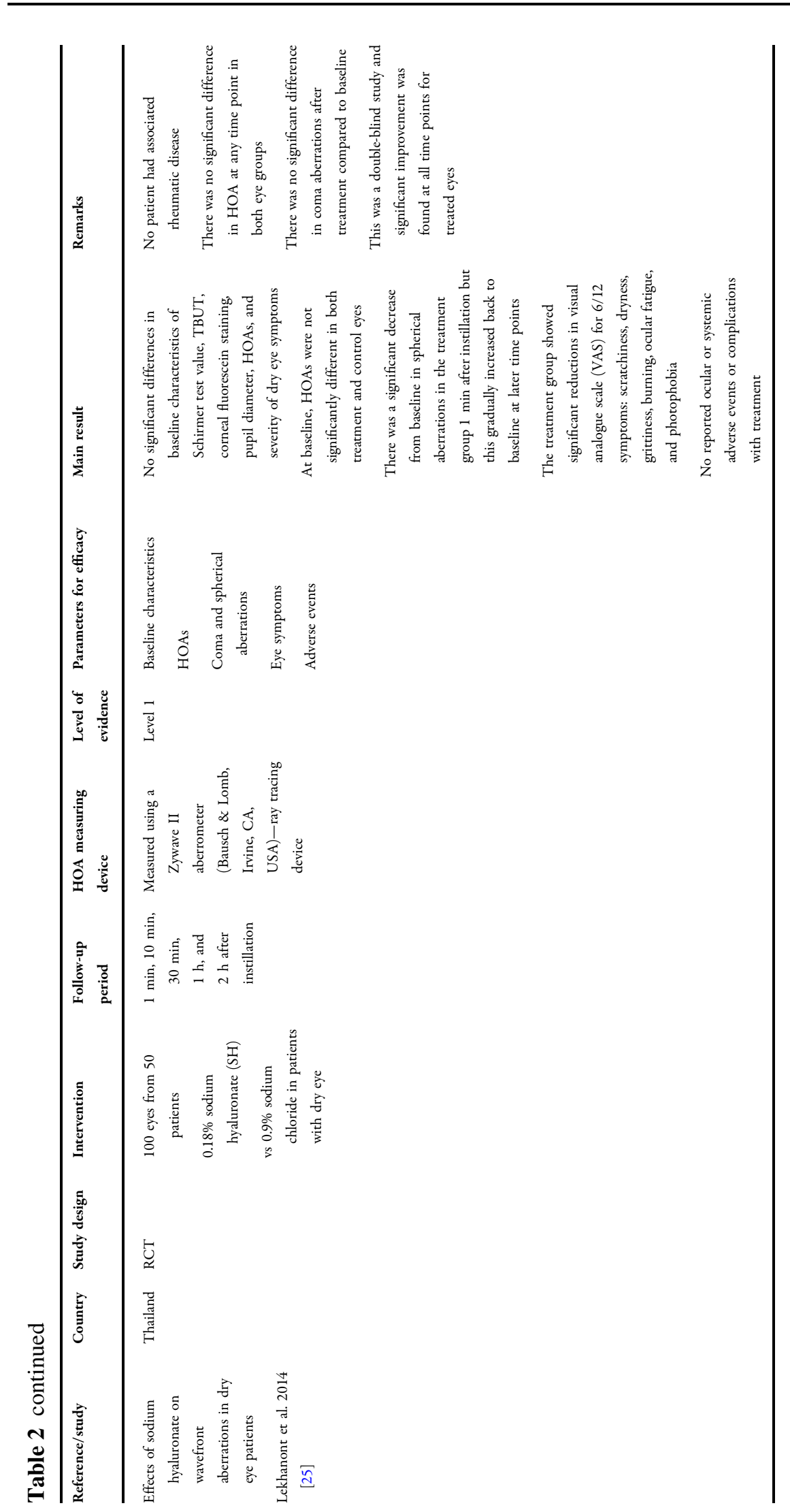




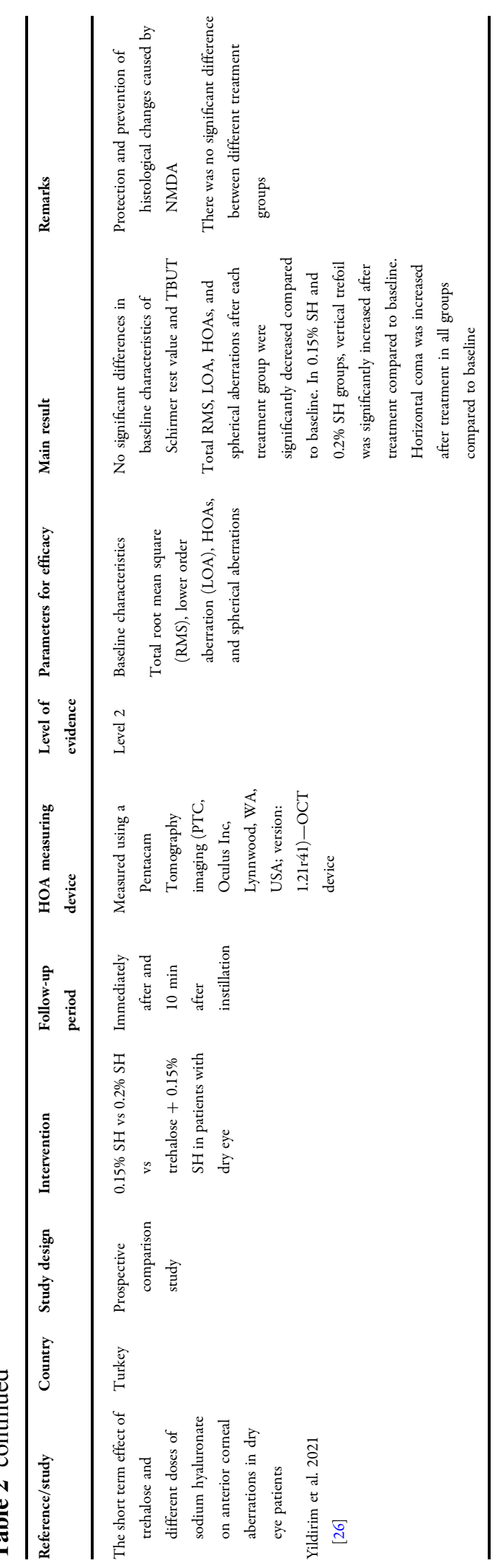




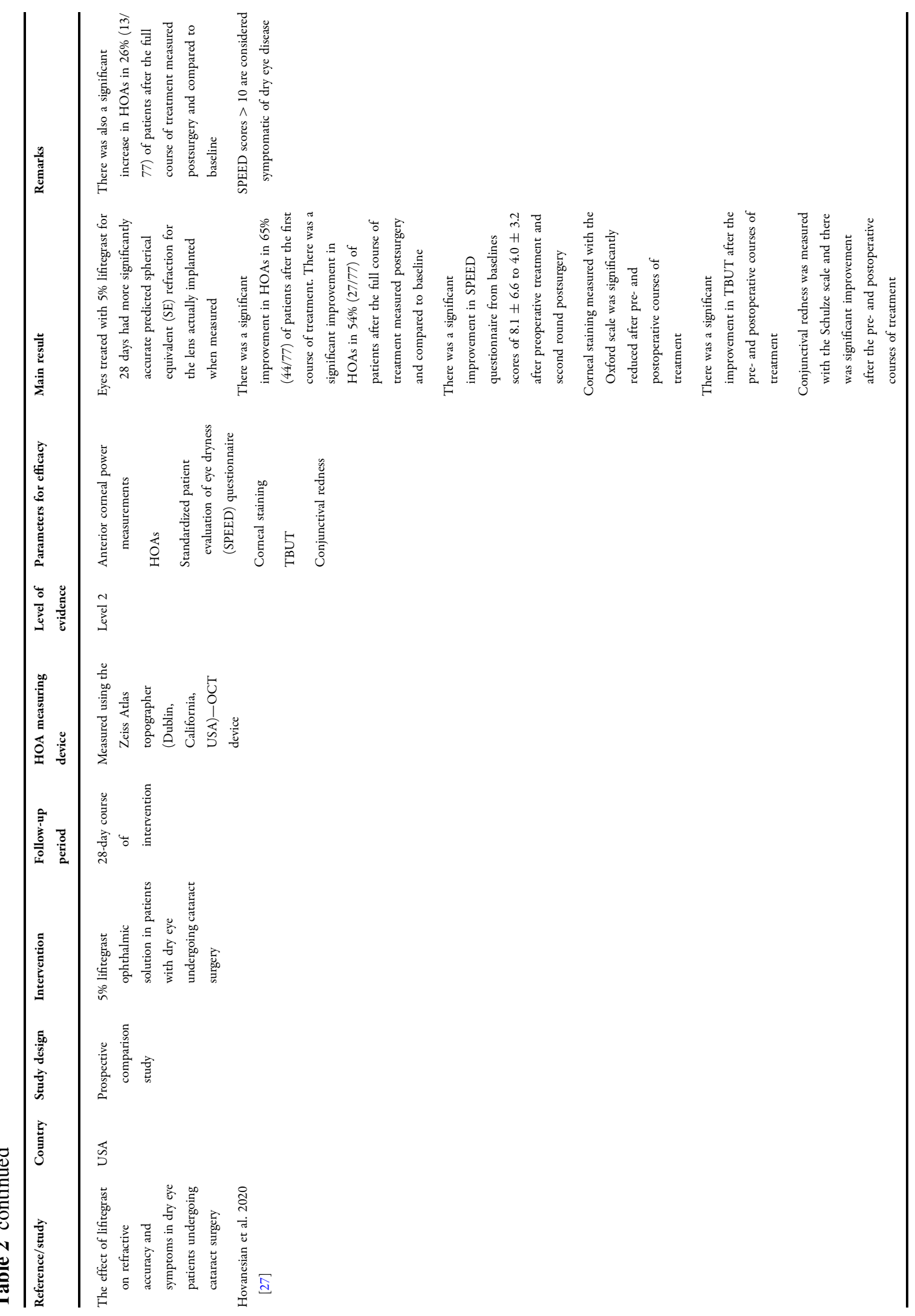




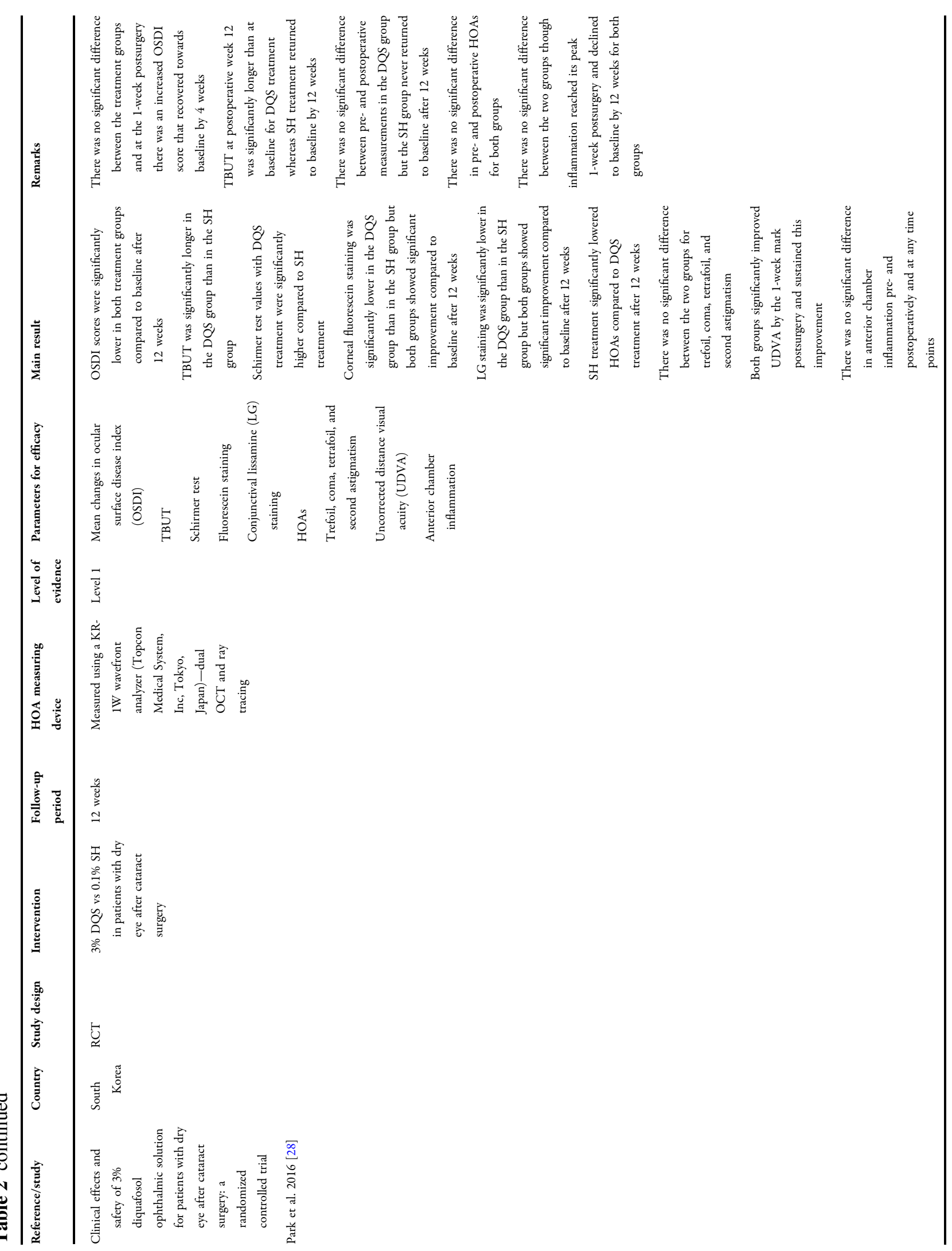




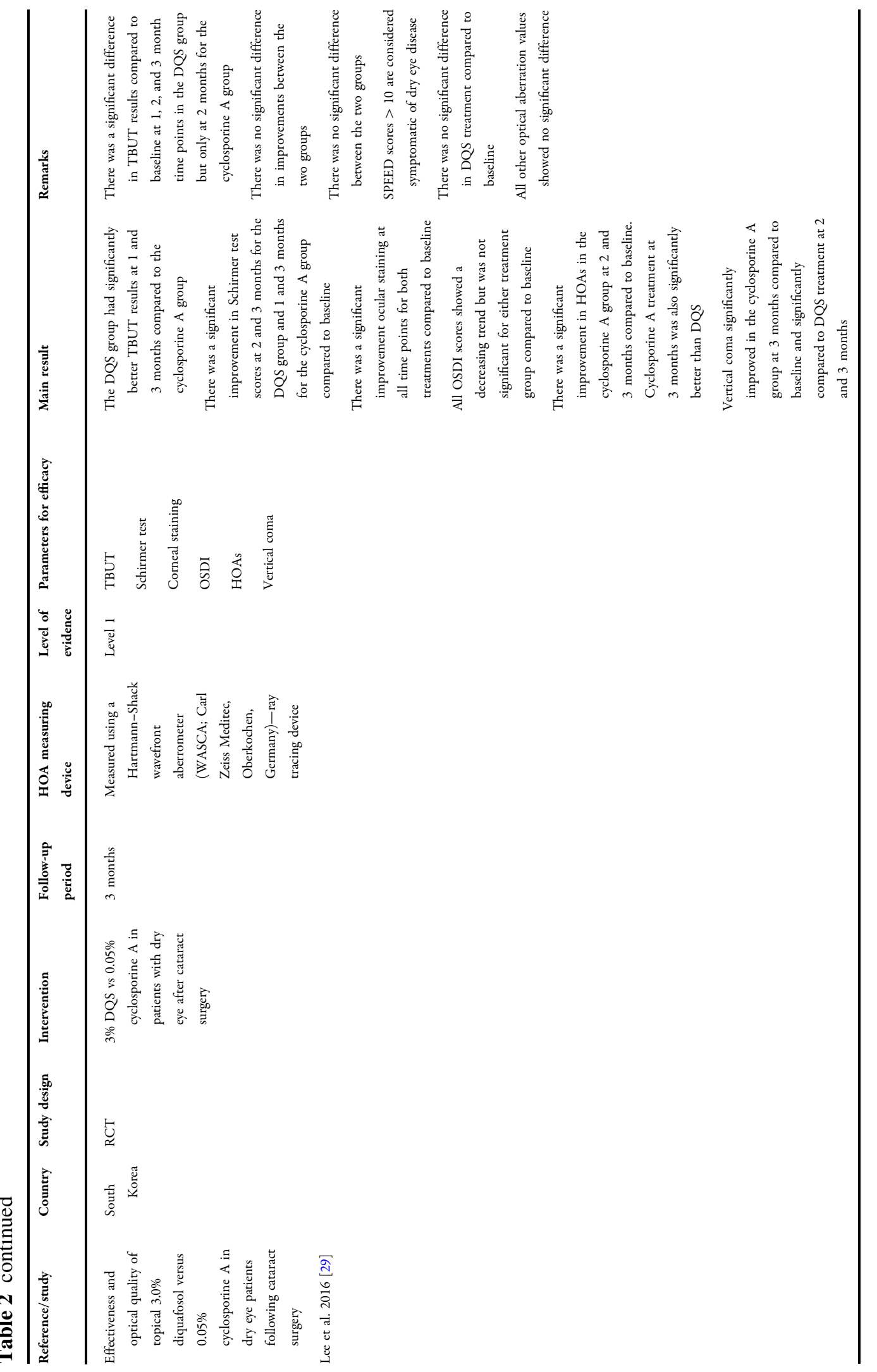




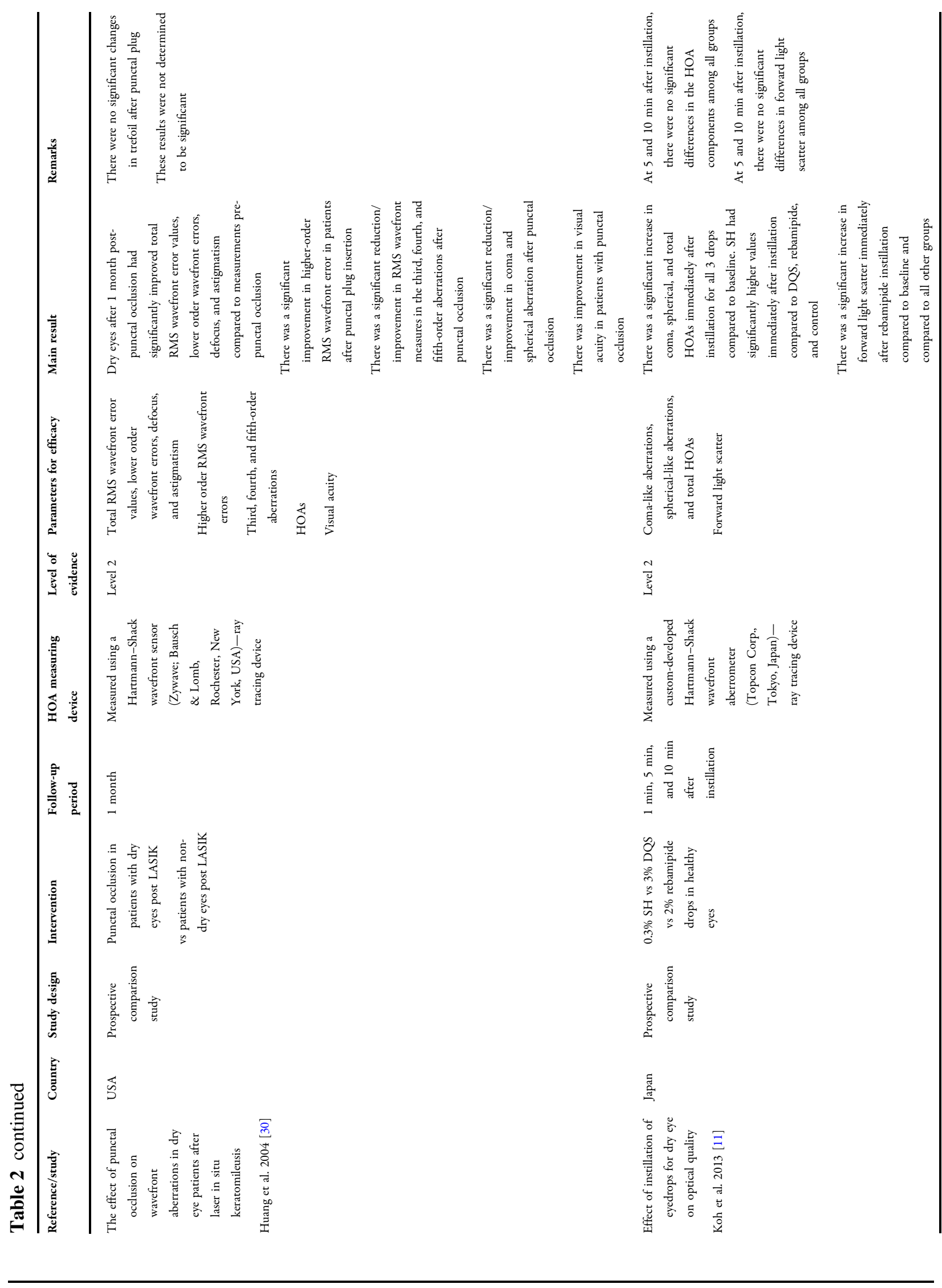




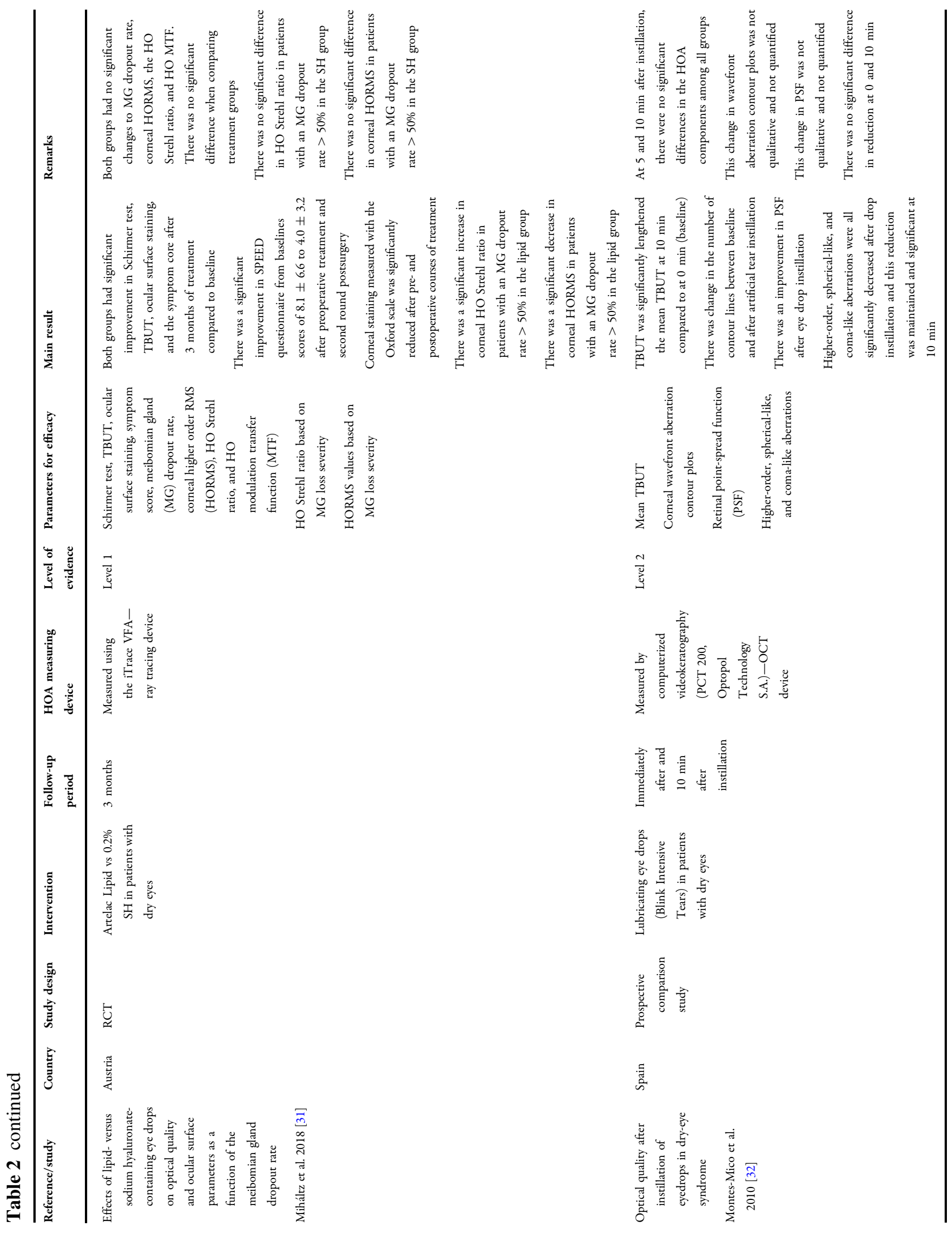




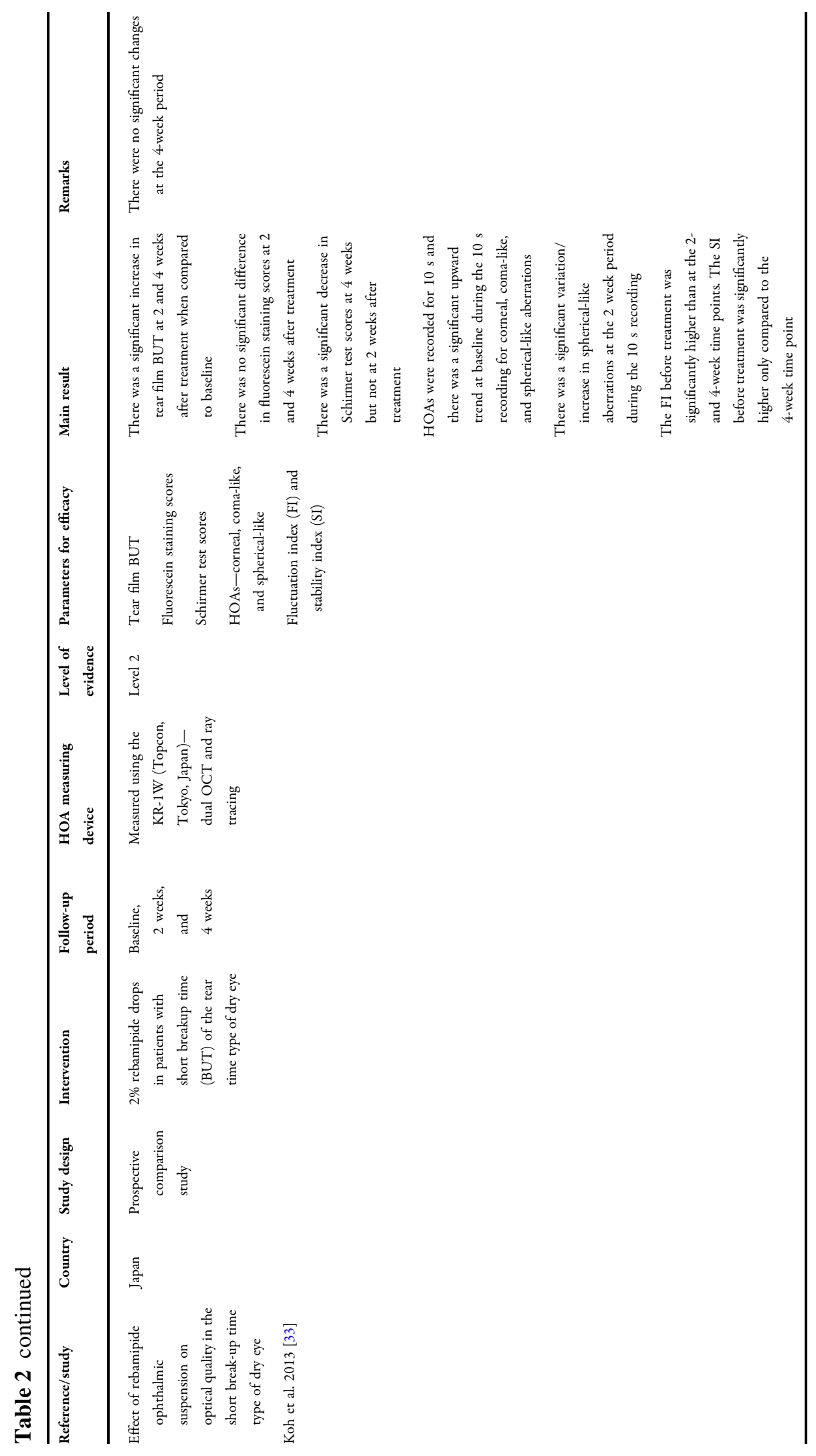




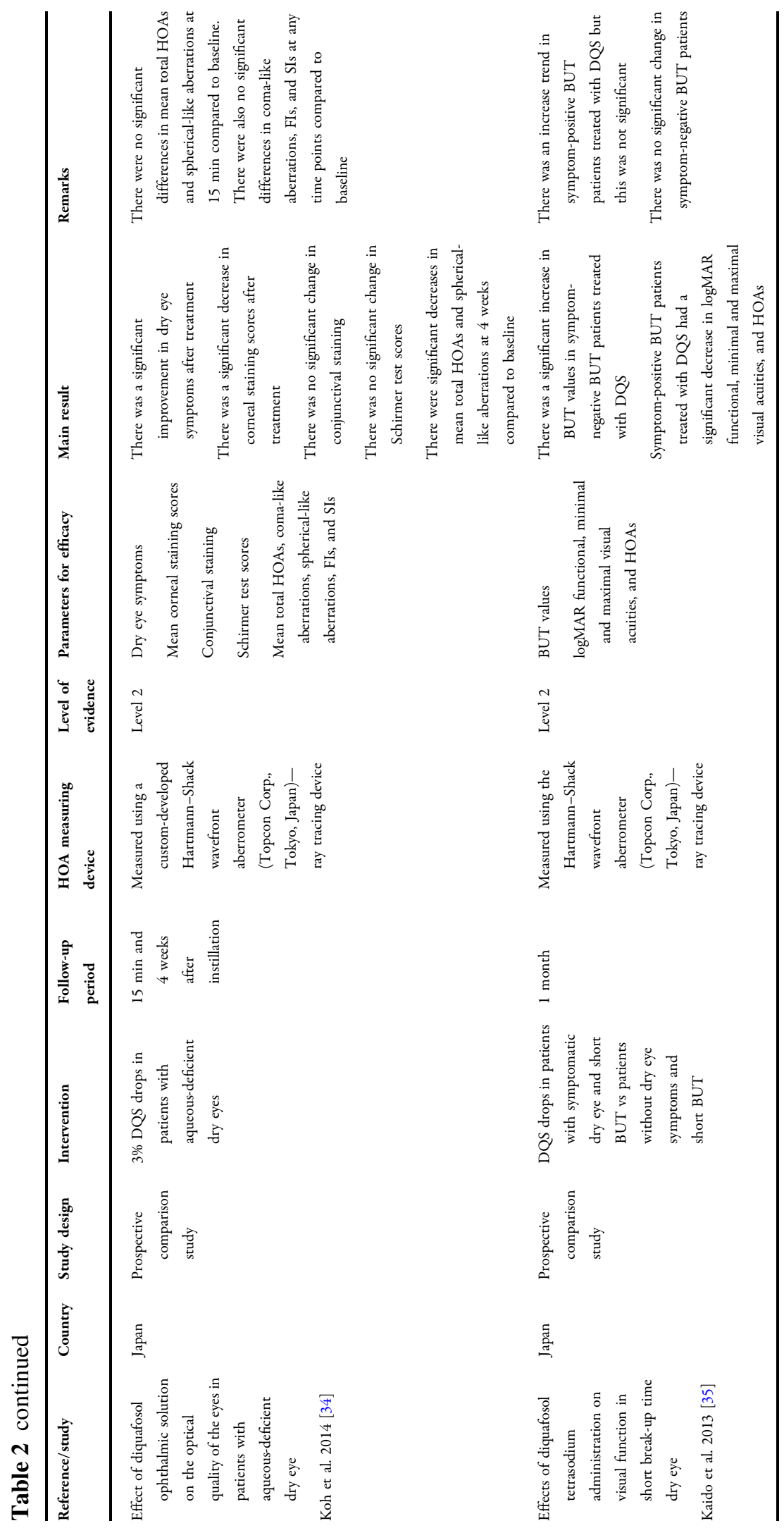




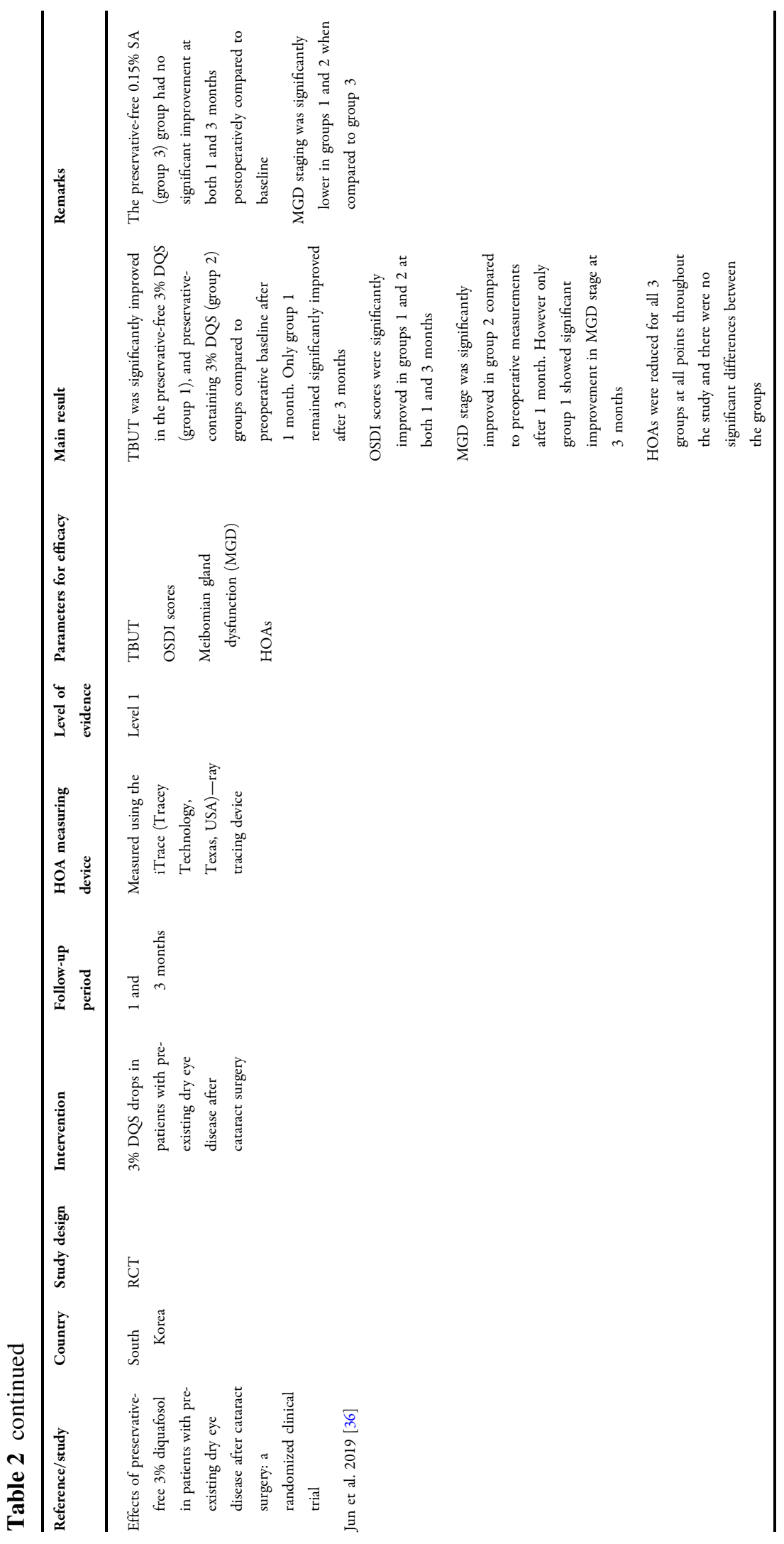


of the tear film compared to areas on the lateral sides of the cornea (left and right) and increased thickening of the tear film at the inferior portion of the cornea. Although differences in vertical and horizontal coma aberrations are expected as a result of gravitational effects on the tear layer and recovery from the effects of lid pressure after blinking, these expected differences were significantly higher in dry eyes compared to normal eyes. These changes in tear film in dry eyes may be responsible for the aberrations and irregularities of the corneal surface.

Another group prospectively assessed serial measurements of HOAs after blinking in patients with dry eyes with or without superficial punctate keratopathy (SPK) [15]. SPK occurs in patients with chronic dry eye disease in which small groups of cells are damaged as a result of inadequate tear production [37]. The total HOAs in the group with SPK were significantly higher than the group without SPK. Similarly, both coma- and spherical-like aberrations were significantly higher in the group with SPK compared to the group without SPK [15]. In the group without SPK, spherical aberrations were significantly higher at 7 and $9 \mathrm{~s}$ compared to $2 \mathrm{~s}$ after the blink. In the group with SPK, spherical aberrations were also higher at $9 \mathrm{~s}$ compared to at 2 and $4 \mathrm{~s}$ after the blink [15]. The increases in spherical-like aberrations over time between blinks found in both groups were also observed in normal eyes in a previous study by the same group [38]. These results suggest that HOAs in dry eye with SPK in the central cornea are higher than those in dry eye without SPK [15].

As mentioned before, laser and refractive surgeries have been shown to cause dry eyes and an increase in HOAs [1, 3, 16]. One group prospectively assessed HOAs in patients who underwent laser subepithelial keratomileusis (LASEK) [16] with and without dry eye. At baseline, the dry eye group had significantly lower tear breakup time (TBUT), Schirmer I test values, and tear clearance rate compared to the non-dry eye group [16]. The ocular surface staining and ocular surface disease index (OSDI) were significantly higher in the dry eye group compared to the non-dry eye group at baseline.
Postoperatively, at $10 \mathrm{~s}$ after blinking, the dry eye group had significantly higher total HOA, coma, and trefoil aberrations compared to the non-dry eye group. The total HOA, coma, and trefoil aberrations were also significantly higher $10 \mathrm{~s}$ after a blink compared to immediately after in the dry eye group [16]. Correlation analysis also revealed that total HOA, coma, and trefoil aberrations had a significantly negative correlation with TBUT while the correlation was significantly positive with OSDI score. These results suggest that additional HOAs caused by tear film instability in patients with dry eye after LASEK may lead to a further reduction in optical quality [16]. Another group prospectively assessed HOAs in patients who underwent laserassisted in situ keratomileusis (LASIK) and compared this to patients with dry eyes and normal eyes [18]. The TBUT was significantly lower for dry eyes compared to both post-LASIK and normal eyes [18]. There was a significant increase in HOAs from post-blink to BUT in patients with normal and dry eyes and a subsequent decrease in HOAs after saline addition [18]. Interestingly, post-LASIK eyes showed no significant differences between the phases [18].

The tear film is subject to dynamic changes and one group assessed the dynamic properties and changes of wavefront aberrations in patients with dry eye and normal eyes [17]. Overall, the group noted that changes in wavefront aberrations and visual acuity is dynamic and variable patient-to-patient (in both dry and normal eyes) [17]. However, the mean instability of total wavefront aberrations and HOAs were significantly greater in patients with dry eye than in normal patients. Similarly, the mean instability of visual acuity was significantly higher in patients with dry eye compared to the normal patients [17]. Interestingly, the instability of visual acuity was dependent on blink rate in the dry eye group, showing that patients with a higher blink rate had more stable visual acuity [17]. This could suggest that patients with dry eye may compensate for tear breakup with increased blinking to maintain stable vision [17]. Another group similarly assessed tear film aberration dynamics in patients with dry eye disease (Sjögren's syndrome) and normal patients [19]. At baseline, 
they found that the patients with dry eye had significantly lower TBUT and Schirmer test values compared to the control group [19]. Meibomian dysfunction and ocular surface disease index (OSDI) scores were significantly higher for patients with dry eye compared to control [19]. After blinking, there was a significant increase in total HOAs, coma, and trefoil aberrations in patients with dry eye [19]. This increase in aberrations was not seen in the control eyes. Total HOAs, coma, and trefoil aberrations were also significantly higher in dry eyes compared to control eyes [19]. The area under the curve of modulation transfer function (aucMTF), where MTF is the measurement of the ability of an optical system to transfer various levels of detail from object to image, was found to be significantly lower in patients with dry eye disease than controls at 7, 8, 9, and $10 \mathrm{~s}$ after blinking [19]. There was also a significantly negative correlation between TBUT and HOA progression index, and a significantly positive correlation between OSDI scores and HOA progression index [19]. The study was able to demonstrate that the time course of tear film HOAs correlates with the clinical examination (TBUT) and patient-reported outcomes (OSDI scores) of dry eye [19].

Dry eye disease has been noted to be more frequent in patients with migraine [20]. One group assessed the impact of dysfunctional tear films and aberrations in patients with migraine and normal eyes [20]. Total aberrations, total HOAs, coma aberrations, spherical aberrations, and objective scatter index (OSI) were significantly higher in normal patients compared to the patients with migraine [20]. Trefoil aberrations and TBUT were not significantly different between the groups [20]. Patients with dry eyes as evaluated with a Schirmer test score $<10 \mathrm{~mm} / 5 \mathrm{~min}$ were excluded [20]. The group's rationale behind this was to prevent overestimation of aberration profile with patients with confirmed dry eyes [20]. On the basis of the results, patients with migraines do not have increased evidence of dry eyes or HOAs.

Another group assessed wavefront aberrations and functional visual acuity as the major causes of visual deterioration in patients with dry eye and Sjögren's syndrome [7]. The group also specifically looked at patients with dry eye and Sjögren's syndrome with and without SPK. The mean visual maintenance ratio (VMR) in the group with SPK was significantly lower than those without SPK and the normal group. The VMR was significantly lower in the group without SPK compared to the normal group [7]. The variation in visual acuity in the group with SPK was significantly higher than in the group without SPK and the normal group. Total HOAs and coma-like aberrations were significantly higher in the SPK group compared to those in the group without SPK and the normal group [7]. Serial changes in HOAs were also tracked and measured over $10 \mathrm{~s}$; however, there were no significant changes in coma, spherical, and total HOAs in any of the groups. A significant negative correlation was found between VMR and both coma and total HOAs [7].

The impact of dry eye disease and functional visual impact in the context of driving was explored by another group [21]. The eyes of patients with dry eye and control patients were compared and total HOAs, coma, and trefoil aberrations varied significantly overtime in patients with dry eye, whereas this variation in aberrations was not found in control patients [21]. To assess the functional impact higher levels of HOA variations have on patients with dry eye, both groups underwent a driving visual performance assessment which required patients to identify targets. Patients with dry eye had a significantly longer response time to identify targets compared to control and were able to detect fewer targets compared to the control group. A significant positive correlation was also found between HOAs and the response time and OSDI symptom score.

In a unique case report, one group reported a woman with Sjögren's syndrome who underwent punctal plug placement to improve her dry eye symptoms [22]. However, despite dry eye symptom improvements, the patient reported epiphora and blurred vision immediately after blinking [22]. The total HOAs during the few seconds after blinking were spiked higher than before the punctal plug insertion [22]. Although these HOAs gradually declined and stabilized, a paradoxical increase in HOAs 
was observed in this patient after punctal plug insertion [22]. The same group also looked into the effects of suppressing blinking on vision quality in 10 patients with no ocular disease but short TBUT [23]. This group was further divided into a normal stable, normal-sawtooth, and short TBUT group [23]. The total HOAs and coma-like aberrations were significantly higher from 5 to $9 \mathrm{~s}$ after a blink compared to immediately after a blink [23]. Spherical-like aberrations were significantly higher from 6 to $9 \mathrm{~s}$ after a blink compared to immediately after a blink [23]. When the three groups were compared in terms of HOAs, fluctuation index (FI), and stability index (SI), the short TBUT group had significantly higher FI and SI compared to the normal stable group. There were no significant differences in FI and SI between the normal-sawtooth and short TBUT group [23].

Overall, the published evidence provides a clear link between dry eye disease and HOAs, in which HOAs are increased with dry eye severity $[4,7,14-16,19,39]$. One of the most important factors to consider is the tear film in the context of dry eyes and HOAs, as many of the studies have demonstrated a significant decrease in TBUT in patients with dry eye $[7,16,18,19]$. Tear film issues should be readily attenuated with lubricant drops, whereas punctate keratitis may not be so readily treated with lubricants. Showing improvements in HOAs after treating dry eyes helps strengthen the relationship and association of HOAs and dry eye severity. Therefore, it is also important to explore whether improvements in dry eye parameters impact HOA severity.

\section{Dry Eye Interventions and Their Effects on Tear Film Metrics and HOA Severity}

Artificial tears are the first-line treatment for dry eye and seem to have a lubrication effect [24]. However, the preservatives in artificial tears have been demonstrated to be detrimental to the ocular surface [24]. Therefore, unpreserved eye drops or alternatives such as liposome sprays are preferred options [24]. The first paper examined dry eye interventions and HOA outcomes using unpreserved Hypromellose drops, a liposome spray solution (Tears Again ${ }^{\mathrm{TM}}$ ), and the combination of the two in normal and dry eyes in an RCT of 48 participants [24]. There was a significant decrease in HOAs immediately after Hypromellose drop instillation compared to baseline in both normal and dry eyes [24]. However, this difference was no longer significant $1 \mathrm{~h}$ after intervention in both eye types. There was no significant difference in the liposome spray or combination intervention at any time point for both eye types [24]. Hypromellose drops significantly increased total aberrations immediately after instillation compared to baseline in both normal and dry eyes but there was a similarly sized and significant reduction $1 \mathrm{~h}$ after intervention in both eye types [24]. Liposome and combination intervention had no significant effect on total aberrations at any time point for both eyes [24]. There were no significant changes in spherical or coma-like aberrations with Hypromellose, liposome, or combination intervention for both eyes [24]. Despite insignificant and short-lived changes in HOAs, both eye type groups reported higher subjective comfort scores after combination treatment, followed by liposome spray, and Hypromellose drops last [24].

Diquafosol sodium ophthalmic solution (DQS) promotes the secretion of water and mucin from conjunctival cells and has been shown to be effective in treating dry eyes, improving visual function, and effective even in post-cataract dry eyes [3]. This group assessed the effects of 3\% DQS and artificial tears (AT) in patients with dry eye after cataract surgery in an RCT of 42 participants [3]. Tear film breakup time was significantly decreased in both DQS and AT groups compared to preoperative TBUT baseline values [3]. After 4 weeks of DQS instillation there was a significant increase in TBUT, but no significant changes in TBUT were seen with AT [3]. Similarly, corneal and conjunctival fluorescein staining was significantly increased in both DQS and AT groups postoperatively but only DQS significantly reduced fluorescein staining after 4 weeks of instillation [3]. There were no significant changes in HOAs after 4 weeks post-cataract surgery in both groups, although DQS treatment stabilized HOA patterns, whereas AT treatment continued to have 
an upward curve pattern [3]. The FI and SI were significantly lower in the DQS group compared to the AT group [3]. DQS treatment was able to stabilize the pattern of HOAs, while AT treatment continued to show increasing sawtooth patterns [3]. Another group assessed the effects of $3 \%$ DQS on 16 patients with aqueous-deficient dry eyes in a prospective comparison study [34]. There were significant improvements in dry eye symptoms and corneal staining after treatment [34]. Schirmer test scores and conjunctival staining were not significantly changed after treatment though [34]. HOAs and spherical aberrations were significantly improved after 4 weeks of instillation when compared to baseline values [34]. However, no changes in coma-like aberrations, FIs, and SIs were seen when compared to baseline values [34]. DQS drops were also assessed in 11 patients with dry eye symptoms and short TBUT compared to patients without dry eye symptoms and short TBUT [35]. There was a significant improvement in TBUT in symptom-negative patients treated with DQS but not with symptom-positive patients [35]. The logMAR functional minimal, maximal, and visual acuities were significantly improved in symptom-positive patients, as were HOAs [35].

Sodium hyaluronate $(\mathrm{SH})$ is a glycosaminoglycan that improves tear film stability and decreases washout from the ocular surface [25]. $\mathrm{SH}$ also binds water and resists dehydration to promote corneal epithelial wound healing [25]. One group assessed the effects of $0.18 \% \mathrm{SH}$ drops and a $0.9 \%$ sodium chloride $(\mathrm{NaCl})$ solution on 50 patients with dry eye in an RCT [25]. At baseline, there were no significant difference in HOAs in both SH- and NaCl-treated eyes [25]. $\mathrm{HOA}$ was measured over $2 \mathrm{~h}$ after $\mathrm{SH}$ or $\mathrm{NaCl}$ instillation and there was no significant difference in HOAs at any time point in both eye groups [25]. There was a significant decrease in spherical aberrations 1 min after $\mathrm{SH}$ instillation, but this gradually increased to baseline levels at later time points [25]. Reported dry eye symptoms were significantly reduced in SH-treated eyes compared to $\mathrm{NaCl}$ control [25]. Another group assessed $\mathrm{SH}$ at differing concentrations, $0.15 \%$ and $0.2 \%$, along with a combination of $0.15 \% \mathrm{SH}$ and trehalose in patients with dry eye in a prospective comparison study [26]. Trehalose provides a hypo-osmolar effect and has positive contributory effects on the corneal surface and cell survival by stabilizing membrane lipids and proteins during desiccation [26]. Trehalose and SH have also been shown to have synergistic action together in the context of dry eyes [26]. The total root mean square (RMS) values of lower order aberrations (LOAs), HOAs, and spherical aberrations were all significantly decreased compared to their respective baseline values for each treatment group $(0.15 \% \mathrm{SH}, 0.2 \% \mathrm{SH}$, and $0.15 \% \mathrm{SH}+$ trehalose) [26]. However, there was no significant difference amongst the treatment groups [26]. Another group compared and assessed the effects of $0.2 \% \mathrm{SH}$ and a lipid-based eye drop (Artelac Lipid) on 30 patients with dry eyes in an RCT [31]. There was a significant improvement in Schirmer test, TBUT, ocular staining, and symptom relief after 3 months in both SHand lipid-treated groups compared to baseline values [31]. There was no significant change in HOAs compared to baseline values [31]. In patients with a meibomian gland (MG) dropout rate greater than $50 \%$, lipid treatment was able to significantly improve higher order Strehl ratio and HOAs compared to baseline [31].

The effect of 3\% DQS compared to $0.1 \% \mathrm{SH}$ in 86 patients with dry eyes after cataract surgery was also assessed in an RCT [28]. Ocular surface disease index (OSDI) was significantly lower in both treatment groups compared to baseline after 12 weeks of treatment, but there was no difference between the treatment groups [28]. TBUT, Schirmer test, and fluorescein staining were significantly improved in the DQS group compared to the SH group [28]. HOAs were significantly lowered and stabilized earlier in the DQS group compared to the SH group, although both groups had no significant difference between pre- and postoperative measurements of HOAs [28]. There was no significant difference in trefoil, coma, tetrafoil, and second astigmatism in both groups after 12 weeks of treatment [28]. Uncorrected distance visual acuity was significantly improved by the 1-week mark postsurgery in both groups and sustained within the 12 -week treatment period [28]. DQS treatment was superior to $\mathrm{SH}$ 
in improving TBUT, changes in HOAs, Schirmer values, and staining scores throughout a 12-week follow-up period [28]. Another study similarly assessed the effects of 3\% DQS (with and without preservatives) compared to $0.15 \%$ SA in 150 patients with pre-existing dry eye disease after cataract surgery in an RCT [36]. TBUT was significantly improved for the preservative-free 3\% DQS and preservativecontaining 3\% DQS group compared to baseline measurements 1 month postoperatively [36]. However, after 3 months, only the preservativefree 3\% DQS group was significantly improved compared to baseline. OSDI scores were significantly improved at all time points throughout the study in both the preservative-free and preservative-containing 3\% DQS groups. MGD stage was significantly reduced in the preservative-containing DQS group at 1 month postoperatively; however at 3 months, only the preservative-free DQS group had significantly improved MGD stage compared to group 3 [36]. HOAs were all significantly improved compared to baseline values for all treatment groups compared to baseline and there were no significant differences between DQS (preservativefree and containing) and $\mathrm{SH}$ [36].

The effects of $3 \%$ DQS, $0.3 \% \mathrm{SH}$, and $2 \%$ rebamipide solution were assessed on 15 healthy patients in a prospective comparison study [11]. Rebamipide stimulates mucous secretion on the ocular surface [11]. There was a significant increase in coma, spherical, and total HOAs immediately after instillation of all three drops (3\% DQS, $0.3 \% \mathrm{SH}$, and $2 \%$ rebamipide) when compared to baseline values [11]. Immediately after instillation, SH led to the highest increase in coma, spherical, and total HOAs compared to DQS, rebamipide, and control [11]. However, 5 and 10 min after instillation, there were no significant differences in coma, spherical, or total HOAs among all groups and compared to baseline [11]. There was a significant increase in forward light scatter immediately after rebamipide instillation compared to baseline and compared to DQS and SH [11]. However, 5 and $10 \mathrm{~min}$ after instillation, there was again no significant difference in forward scatter among the groups and compared to baseline [11]. The same group assessed the effects of $2 \%$ rebamipide on 16 patients with short TBUT type of dry eye in a prospective comparison study [33]. TBUT was significantly increased at the measured 2- and 4-week time points after treatment when compared to baseline [33]. Fluorescein staining and Schirmer test scores were not significantly different at 2 and 4 weeks after treatment [33]. During 10-s recordings of HOAs, there was a significant upward trend at baseline for coma, spherical, and total HOAs [33]. At the 2-week time point, only spherical aberrations had a significant increase in during the 10-s recording period/post-blink change [33]. At 4 weeks, there were no significant changes in coma, spherical, or total HOAs, indicating stability in HOAs [33]. The FI was significantly improved at the 2- and 4-week periods, whereas SI was significantly improved only compared to the 4 -week period [33].

Cyclosporine A is a calcineurin inhibitor and potent immunomodulatory agent that inhibits T cell activation and reduces surface inflammation in the context and usage as an eye drop [29]. This group assessed the effects of 3\% DQS and $0.05 \%$ cyclosporine A (Restasis $^{\circledR}$ ) in 40 patients with dry eye after cataract surgery in an RCT [29]. DQS significantly improved TBUT at 1 and 3 months compared to the cyclosporine $\mathrm{A}$ group [29]. Schirmer test scores and corneal staining were significantly improved for both treatment groups, but there was no difference between the groups [29]. HOAs were significantly improved with cyclosporine at 2 and 3 months compared to baseline values and significantly better than DQS at 3 months after treatment [29]. Vertical coma was also significantly improved in the cyclosporine A group at 3 months compared to baseline and at both 2 and 3 months when compared to DQS [29]. DQS was more effective at increasing tear secretions and improving TBUT, but cyclosporine A was more effective at improving HOAs [29].

Lifitegrast $5 \%$ is a pharmacologic eye drop that inhibits the inflammation associated with dry eye disease by blocking $\mathrm{T}$ cell adhesion and migration through inhibition of lymphocyte function-associated antigen 1 (LFA-1) to intercellular adhesion molecule 1 (ICAM-1) binding $[27,40]$. Lifitegrast is approved by the US Food 
and Drug Administration for treating both the signs and symptoms of dry eye disease $[27,40]$. One group assessed the effects of 5\% lifitegrast solution in 100 patients with dry eyes undergoing cataract surgery in a prospective comparison study [27]. Eyes that were treated with lifitegrast for 28 days had significantly more accurate predicted spherical equivalent refraction for the lens actually implanted [27]. There was also a significant improvement and reduction in HOAs in 65\% (44/77) of patients after the first course of treatment and a significant improvement in HOAs in 54\% (27/77) of patients after the full course of treatment when compared to baseline values [27]. Corneal staining, TBUT, and conjunctival redness were all significantly improved after treatment with lifitegrast [27]. Standardized patient evaluation of eye dryness questionnaire also revealed a significant improvement in dry eye symptoms after lifitegrast treatment [27].

Punctal plug procedures are another intervention for dry eyes that block the puncta which increases tear fluid accumulation, thus keeping the tear film intact [13]. One group assessed the effect of punctal plugs in eight patients with dry eye after LASIK in a prospective comparison study [30]. Patients with dry eye after 1 month of post-punctal occlusion had significantly improved RMS error values, lower order wavefront errors, defocus, and astigmatism compared to pre-punctal measurements [30]. There was also a significant improvement in the HOAs, spherical, and coma aberrations after punctal plug insertion [30].

One group assessed the effects of lubricating eye drops (Blink Intensive Tears) on 20 patients with dry eyes in a prospective comparison study [32]. The TBUT was significantly lengthened at 10 min after instillation when compared to baseline [32]. Spherical, coma, and HOAs were significantly decreased immediately after instillation of eye drops and were significantly maintained after $10 \mathrm{~min}$ [32].

\section{DISCUSSION}

As mentioned before, several studies have demonstrated that disruptions in the tear film caused by dry eye increase HOAs compared to normal eyes which results in impaired visual function and optical quality [4, 6-9]. Of the 12 papers directly looking at dry eyes and HOA outcomes, eight have definitively shown that there is a significant difference and increase in HOAs in patients with dry eyes $[4,7,14-17,19,21]$ and that with increasing dry eye severity, defined as patients with SPK, there is significant increase in HOAs as well $[7,15]$. The other five papers had differing results in supporting the association of dry eyes and HOAs. In one paper, although the differences in HOAs between normal and dry eyes were not assessed, TBUT was significantly less in dry eyes compared to normal eyes and total HOAs were decreased after saline drops in both patients with normal and dry eyes [18]. Patients with migraines are reported to have more frequent dry eye disease [20] and this group was able to demonstrate that coma, spherical, and total HOAs were significantly higher in their control group compared to the migraine group [20]. This group specifically excluded patients with actual dry eye disease so as not to overestimate their measured parameters though [20]. The last two case reports by the same group showed an opposite phenomenon where HOAs were increased after blinking in post-punctal plug placement patients and in patients with short TBUT [22, 23]. HOAs tend to increase in normal patients and patients with dry eye post blink due to disruption and changes in the tear film $[4,8,22,41]$. Before the tear film breaks up, the difference in tear film thickness of the thinner superior and thicker inferior cornea may lead to delayed wavefronts inferiorly and increased coma-like aberrations due to the longer optical path in the inferior tear film compared to the thinner superior tear film [22]. Similarly, punctal occlusion in some cases of mild dry eye may cause visual impairment due to the post-blink movement of excessive tear volume [22].

A significant negative correlation between HOAs and TBUT was also demonstrated, providing further evidence that as TBUT decreases, and consequently dry eye severity increases, HOAs increases $[16,19]$. 
As the tear film and TBUT are crucial factors in dry eye disease and HOAs, tear film issues should be readily attenuated with interventions to improve TBUT or help stimulate lipid and aqueous secretions. Therefore, showing improvements in HOAs after treating dry eyes would help strengthen the relationship of HOAs and dry eye severity. From the 15 papers looking at dry eye interventions and HOA outcomes, 12 have shown improvements in HOAs to varying degrees [24-32, 34-36]. In particular, some papers did reveal improvements in HOAs but only for a short time period immediately after instillation of the intervention [25], or revealed an improvement in total HOAs but an increase in vertical trefoil and horizontal coma aberrations [26]. The variation in results can be a result of many factors, including the variation in patient dry eye severity as well as the type of eye drop and intervention used. The viscosity of eye drops plays an important role in the impact of aberrations and has been shown to impact aberration parameters [26, 33]. The type of intervention used also plays a role as one study was able to show that $\mathrm{SH}$ was able to significantly improve TBUT over DQS, but DQS was able to improve HOAs significantly more than $\mathrm{SH}$ [28]. Whereas, another study demonstrated that DQS and SH were both able to significantly improve HOAs without any significant difference between the two [36]. Another paper demonstrated that DQS significantly improved TBUT over cyclosporine A, but cyclosporine A was able to significantly improve HOAs over DQS [29]. In addition, DQS did not significantly change HOA outcomes and the group postulated that this may be due to $3 \%$ DQS causing excessive secretion of fluid that paradoxically disturbs tear film stability [29]. Related to the viscosity and makeup of the eye drops are the morphological changes and patterns that certain eye drops and artificial tears can produce on the ocular surface, which may be attribute to the variation in clinical success seen [42]. The turnover of tear film components, in particular the lipid components, plays an important role in the effectiveness of eye drops and is an important aspect to consider when utilizing lipid-based eye drop treatments [43].
The variation in dry eye disease in patients also played an important role when looking at HOA outcomes. For instance, in one study that assessed the effects of SH in their dry eye groups, HOAs were not significantly changed after instillation [25] while other studies have shown that SH improved HOAs [32, 44]. The researchers attributed this to their population group having both aqueous tear-deficient and evaporative dry eye with moderate to severe dry eye grading with some cases of SPK [25], compared to the patients with more mild dry eye in previous studies [32, 41].

One of the limitations of our paper includes the possibility that not all primary articles looking into patients with dry eye and HOAs were captured. However, we have tried to overcome these limitations by utilizing a very broad initial search strategy as mentioned in our "Methods" section and curated for relevant papers on the basis of Fig. 1.

Overall, there is evidence to show that an association between dry eyes and HOAs exists and that treating dry eye disease through interventions including eye drops, secretagogues, and punctal plugs can improve HOA outcomes. As dry eye disease is one of the most common ocular disorders and complications from many common laser and refractive surgeries, the clinical implications of improving HOAs are an important area of study. Factors such as patient variation in dry eye disease and severity as well as eye drop type and viscosity are a few of the many areas of study that need to be further elucidated for better clinical application.

\section{CONCLUSION}

On the basis of this systematically approached literature review, dry eyes and HOAs have a clear association with the tear film being one of the most important factors in this relationship between the two. Improvements in HOAs with dry eye treatment interventions such as artificial tears, secretagogues, and punctal plugs provide further evidence on this relationship between dry eyes and HOAs. However, there is still much more work to be done in the realm of 
clinical application as dry eye interventions vary depending on many factors including patient severity, intervention type, eye drop viscosity, and the mechanism of action of intervention. Therefore, although improving HOAs seems to be a viable clinical approach, further evidence and work are required for improved clinical application and outcomes.

\section{ACKNOWLEDGEMENTS}

Funding. No funding or sponsorship was received for this study or publication of this article. The journal's Rapid Service Fee was funded by the authors.

Authorship. All named authors meet the International Committee of Medical Journal Editors (ICMJE) criteria for authorship for this article, take responsibility for the integrity of the work as a whole, and have given their approval for this version to be published.

Author Contributions. Jess Rhee and Kendrick Co Shih were involved in study design, data collection, data analysis, manuscript writing and editing. Tommy Chung-Yan Chan, Sharon Shu-Wan Chow, Antonio Di Zazzo, Takenori Inomata and Louis Tong were involved in data collection, data analysis, manuscript writing and editing.

Disclosures. The following authors confirm that they have no relevant financial disclosures to declare: Jess Rhee, Tommy Chung-Yan Chan, Sharon Shu-Wan Chow, Antonio Di Zazzo, Takenori Inomata, Kendrick Co Shih, Louis Tong.

Compliance with Ethics Guidelines. This article is based on previously conducted studies and does not contain any new studies with human participants or animals performed by any of the authors.

Data Availability. This is a review article and, as such, does not generate new data. The authors agree to make all materials, data and associated protocols promptly available to readers without undue qualifications in material transfer agreements.

Open Access. This article is licensed under a Creative Commons Attribution-NonCommercial 4.0 International License, which permits any non-commercial use, sharing, adaptation, distribution and reproduction in any medium or format, as long as you give appropriate credit to the original author(s) and the source, provide a link to the Creative Commons licence, and indicate if changes were made. The images or other third party material in this article are included in the article's Creative Commons licence, unless indicated otherwise in a credit line to the material. If material is not included in the article's Creative Commons licence and your intended use is not permitted by statutory regulation or exceeds the permitted use, you will need to obtain permission directly from the copyright holder. To view a copy of this licence, visit http:// creativecommons.org/licenses/by-nc/4.0/.

\section{REFERENCES}

1. Charman WN. Wavefront technology: past, present and future. Cont Lens Anterior Eye. 2005;28:75-92. https://doi.org/10.1016/j.clae.2005.02.003.

2. Suliman A, Rubin A. A review of higher order aberrations of the human eye. Afr Vis Eye Heal. 2019;78:1. https://doi.org/10.4102/aveh.v78i1.501.

3. Inoue $\mathrm{Y}$, Ochi S. Effects of 3\% diquafosol sodium ophthalmic solution on higher-order aberrations in patients diagnosed with dry eye after cataract surgery. Clin Ophthalmol. 2017;11:87-93. https://doi. org/10.2147/OPTH.S122542.

4. Montés-Micó R, Cáliz A, Alió JL. Wavefront analysis of higher order aberrations in dry eye patients. J Refract Surg. 2004;20:243-7.

5. Koh S. Irregular astigmatism and higher-order aberrations in eyes with dry eye disease. Invest Ophthalmol Vis Sci. 2018;59:DES36-40. https://doi. org/10.1167/iovs.17-23500.

6. Goto E, Yagi Y, Matsumoto Y, Tsubota K. Impaired functional visual acuity of dry eye patients. Am J 
Ophthalmol. 2002;133:181-6. https://doi.org/10. 1016/S0002-9394(01)01365-4.

7. Kaido M, Matsumoto Y, Shigeno Y, Ishida R, Dogru $\mathrm{M}$, Tsubota K. Corneal fluorescein staining correlates with visual function in dry eye patients. Invest Ophthalmol Vis Sci. 2011;52:9516-22. https://doi. org/10.1167/iovs.11-8412.

8. Montés-Micó R, Alió JL, Muñoz G, Pérez-Santonja JJ, Charman WN. Postblink changes in total and corneal ocular aberrations. Ophthalmology. 2004;111:758-67. https://doi.org/10.1016/j. ophtha.2003.06.027.

9. Zhang Y, Potvin R, Gong L. A study of the shortterm effect of artificial tears on contrast sensitivity in patients with Sjögren's syndrome. Invest Ophthalmol Vis Sci. 2013;54:7977-82. https://doi.org/ $10.1167 /$ iovs.13-11798.

10. Lu N, Lin F, Huang Z, He Q, Han W. Changes of corneal wavefront aberrations in dry eye patients after treatment with artificial lubricant drops. J Ophthalmol. 2016. https://doi.org/10.1155/2016/ 1342056.

11. Koh S, Maeda N, Ikeda C, et al. Effect of instillation of eyedrops for dry eye on optical quality. Invest Ophthalmol Vis Sci. 2013;54:4927-33. https://doi. org/10.1167/iovs.13-12409.

12. Diaz-Valle D, Arriola-Villalobos P, García-Vidal SE, et al. Effect of lubricating eyedrops on ocular light scattering as a measure of vision quality in patients with dry eye. J Cataract Refract Surg. 2012;38: 1192-7. https://doi.org/10.1016/j.jcrs.2012.02.040.

13. Yellepeddi VK, Sheshala R, McMillan H, Gujral C, Jones D, Raghu Raj Singh T. Punctal plug: a medical device to treat dry eye syndrome and for sustained drug delivery to the eye. Drug Discov Today. 2015;20:884-9. https://doi.org/10.1016/j.drudis. 2015.01.013.

14. Shimizu E, Aketa N, Yazu H, et al. Corneal higherorder aberrations in eyes with chronic ocular graftversus-host disease. Ocul Surf. 2020;18:98-107. https://doi.org/10.1016/j.jtos.2019.10.005.

15. Koh S, Maeda N, Hirohara Y, et al. Serial measurements of higher-order aberrations after blinking in patients with dry eye. Invest Ophthalmol Vis Sci. 2008;49:133-8. https://doi.org/10.1167/iovs.070762 .

16. Jung HH, Ji YS, Oh HJ, Yoon KC. Higher order aberrations of the corneal surface after laser subepithelial keratomileusis. Korean J Ophthalmol. 2014;28:285-91. https://doi.org/10.3341/kjo.2014. 28.4.285.
17. Wang $Y, X u$ J, Sun $X$, Chu R, Zhuang H, He JC. Dynamic wavefront aberrations and visual acuity in normal and dry eyes. Clin Exp Optom. 2009;92: 267-73. https://doi.org/10.1111/j.1444-0938.2009. 00354.x.

18. Lin Y-Y, Carrel H, Wang I-J, Lin P-J, Hu F-R. Effect of tear film break-up on higher order aberrations of the anterior cornea in normal, dry, and post-LASIK eyes. J Refract Surg. 2005;21:S525-9.

19. Denoyer A, Rabut G, Baudouin C. Tear film aberration dynamics and vision-related quality of life in patients with dry eye disease. Ophthalmology. 2012;119:1811-8. https://doi.org/10.1016/j. ophtha.2012.03.004.

20. Shetty R, Deshpande K, Jayadev C, et al. The impact of dysfunctional tear films and optical aberrations on chronic migraine. Eye Vis (Lond). 2017;4:4. https://doi.org/10.1186/s40662-017-0070-1.

21. Deschamps N, Ricaud X, Rabut G, Labbé A, Baudouin $C$, Denoyer A. The impact of dry eye disease on visual performance while driving. Am J Ophthalmol. 2013;156:184-189.e3. https://doi.org/10. 1016/j.ajo.2013.02.019.

22. Koh S, Maeda N, Ninomiya S, et al. Paradoxical increase of visual impairment with punctal occlusion in a patient with mild dry eye. J Cataract Refract Surg. 2006;32:689-91. https://doi.org/10. 1016/j.jcrs.2006.01.021.

23. Koh S, Maeda N, Hori Y, et al. Effects of suppression of blinking on quality of vision in borderline cases of evaporative dry eye. Cornea. 2008;27:275-8. https://doi.org/10.1097/ICO.0b013e31815be9c8.

24. McGinnigle S, Eperjesi F, Naroo SA. A preliminary investigation into the effects of ocular lubricants on higher order aberrations in normal and dry eye subjects. Cont Lens Anterior Eye. 2014;37:106-10. https://doi.org/10.1016/j.clae.2013.08.156.

25. Lekhanont K, Chuckpaiwong V, Vongthongsri A, Sangiampornpanit T. Effects of sodium hyaluronate on wavefront aberrations in dry eye patients. Optom Vis Sci Off Publ Am Acad Optom. 2014;91: 39-46. https://doi.org/10.1097/OPX. 0000000000000101.

26. Yildirim Y, Ozsaygili C, Kucuk B. The short term effect of trehalose and different doses of sodium hyaluronate on anterior corneal aberrations in dry eye patients. Cutan Ocul Toxicol. 2021;40:14-20. https://doi.org/10.1080/15569527.2020.1861001.

27. Hovanesian J, Epitropoulos A, Donnenfeld ED, Holladay JT. The effect of lifitegrast on refractive accuracy and symptoms in dry eye patients 
undergoing cataract surgery. Clin Ophthalmol. 2020;14:2709-16. https://doi.org/10.2147/OPTH. S264520.

28. Park DH, Chung JK, Seo DR, Lee SJ. Clinical effects and safety of $3 \%$ diquafosol ophthalmic solution for patients with dry eye after cataract surgery: a randomized controlled trial. Am J Ophthalmol. 2016;163:122-131.e2. https://doi.org/10.1016/j.ajo. 2015.12.002.

29. Lee JH, Song IS, Kim KL, Yoon SY. Effectiveness and optical quality of topical 3.0\% diquafosol versus 0 . $05 \%$ cyclosporine $\mathrm{A}$ in dry eye patients following cataract surgery. J Ophthalmol. 2016;2016: 8150757. https://doi.org/10.1155/2016/8150757.

30. Huang B, Mirza MA, Qazi MA, Pepose JS. The effect of punctal occlusion on wavefront aberrations in dry eye patients after laser in situ keratomileusis. Am J Ophthalmol. 2004;137:52-61. https://doi.org/ 10.1016/s0002-9394(03)00903-6.

31. Miháltz K, Faschinger EM, Vécsei-Marlovits PV. Effects of lipid- versus sodium hyaluronate-containing eye drops on optical quality and ocular surface parameters as a function of the meibomian gland dropout rate. Cornea. 2018;37:886-92. https://doi.org/10.1097/ICO.0000000000001523.

32. Montés-Micó R, Cerviño A, Ferrer-Blasco T, GarcíaLázaro S, Ortí-Navarro S. Optical quality after instillation of eyedrops in dry-eye syndrome. J Cataract Refract Surg. 2010;36:935-40. https://doi. org/10.1016/j.jcrs.2009.12.044.

33. Koh S, Inoue Y, Sugmimoto T, Maeda N, Nishida K. Effect of rebamipide ophthalmic suspension on optical quality in the short break-up time type of dry eye. Cornea. 2013;32:1219-23. https://doi.org/ 10.1097/ICO.0b013e318294f97e.

34. Koh S, Maeda N, Ikeda C, et al. Effect of diquafosol ophthalmic solution on the optical quality of the eyes in patients with aqueous-deficient dry eye. Acta Ophthalmol. 2014;92:e671-5. https://doi.org/ 10.1111/aos.12443.

35. Kaido M, Uchino M, Kojima T, Dogru M, Tsubota K. Effects of diquafosol tetrasodium administration on visual function in short break-up time dry eye. J Ocul Pharmacol Ther. 2013;29:595-603. https:// doi.org/10.1089/jop.2012.0246.
36. Jun I, Choi S, Lee GY, et al. Effects of preservativefree $3 \%$ diquafosol in patients with pre-existing dry eye disease after cataract surgery: a randomized clinical trial. Sci Rep. 2019;9:12659. https://doi.org/ 10.1038/s41598-019-49159-0.

37. Su T, Ting P, Chang S, Chen D. Superficial punctate keratitis grading for dry eye screening using deep convolutional neural networks. IEEE Sens J. 2020;20:1672-8. https://doi.org/10.1109/JSEN. 2019.2948576.

38. Koh S, Maeda N, Hirohara Y, et al. Serial measurements of higher-order aberrations after blinking in normal subjects. Invest Ophthalmol Vis Sci. 2006;47:3318-24. https://doi.org/10.1167/iovs.060018.

39. Wang $\mathrm{H}, \mathrm{Hu} \mathrm{H}$, Zhang $\mathrm{Q}$, et al. Dynamic transcriptomes of human myeloid leukemia cells. Genomics. 2013;102:250-6. https://doi.org/10. 1016/j.ygeno.2013.06.004.

40. Nichols KK, Donnenfeld ED, Karpecki PM, et al. Safety and tolerability of lifitegrast ophthalmic solution 5.0\%: pooled analysis of five randomized controlled trials in dry eye disease. Eur J Ophthalmol. 2019;29:394-401. https://doi.org/10.1177/ 1120672118791936.

41. Koh S, Maeda N, Kuroda T, et al. Effect of tear film break-up on higher-order aberrations measured with wavefront sensor. Am J Ophthalmol. 2002;134:115-7. https://doi.org/10.1016/S00029394(02)01430-7.

42. Napoli PE, Satta GM, Coronella F, Fossarello M. Spectral-domain optical coherence tomography study on dynamic changes of human tears after instillation of artificial tears. Invest Ophthalmol Vis Sci. 2014;55:4533-40. https://doi.org/10.1167/iovs. 14-14666.

43. Napoli PE, Coronella F, Satta GM, Fossarello M. A novel technique of contrast-enhanced optical coherence tomography imaging in evaluation of clearance of lipids in human tears. PLoS ONE. 2014;9:e109843.

44. Montés-Micó R, Cáliz A, Alió JL. Changes in ocular aberrations after instillation of artificial tears in dryeye patients. J Cataract Refract Surg. 2004;30: 1649-52. https://doi.org/10.1016/j.jcrs.2004.02. 041. 